\title{
Spectral Empirical Orthogonal Function Analysis of Weather and Climate Data
}

\author{
OLIVER T. SCHMIDT \\ University of California San Diego, La Jolla, California \\ Gianmarco Mengaldo \\ California Institute of Technology, Pasadena, California \\ GiANPAOLO BALSAMO AND NiLS P. WEDI \\ European Centre for Medium-Range Weather Forecasts, Reading, United Kingdom
}

(Manuscript received 26 September 2018, in final form 26 April 2019)

\begin{abstract}
We apply spectral empirical orthogonal function (SEOF) analysis to educe climate patterns as dominant spatiotemporal modes of variability from reanalysis data. SEOF is a frequency-domain variant of standard empirical orthogonal function (EOF) analysis, and computes modes that represent the statistically most relevant and persistent patterns from an eigendecomposition of the estimated cross-spectral density matrix (CSD). The spectral estimation step distinguishes the approach from other frequency-domain EOF methods based on a single realization of the Fourier transform, and results in a number of desirable mathematical properties: at each frequency, SEOF yields a set of orthogonal modes that are optimally ranked in terms of variance in the $L_{2}$ sense, and that are coherent in both space and time by construction. We discuss the differences between SEOF and other competing approaches, as well as its relation to dynamical modes of stochastically forced, nonnormal linear dynamical systems. The method is applied to ERA-Interim and ERA-20C reanalysis data, demonstrating its ability to identify a number of well-known spatiotemporal coherent meteorological patterns and teleconnections, including the Madden-Julian oscillation (MJO), the quasi-biennial oscillation (QBO), and the El Niño-Southern Oscillation (ENSO) (i.e., a range of phenomena reoccurring with average periods ranging from months to many years). In addition to two-dimensional univariate analyses of surface data, we give examples of multivariate and three-dimensional meteorological patterns that illustrate how this technique can systematically identify coherent structures from different sets of data. The MATLAB code used to compute the results presented in this study, including the download scripts for the reanalysis data, is freely available online.
\end{abstract}

\section{Introduction}

Coherent spatiotemporal structures are pervasive in nature and their systematic identification can help characterize the behavior of complex systems and facilitate their modeling. Earth's atmosphere and its complex coupling to other Earth system components such as ocean, sea ice, and land surfaces, exhibits many of these structures. Several statistically persistent patterns associated with the intrinsic variability of the Earth system exist, and these usually play a critical role in extendedrange and seasonal forecasting of both regional and

Corresponding author: Oliver T. Schmidt, oschmidt@ucsd.edu global-scale weather phenomena (see, e.g., Palmer and Anderson 1994; Wu et al. 2009; Bierkens and Van Beek 2009). The representation and understanding of these coherent structures through a reduced and compact set of predictors can enhance the predictability of the Earth system and ultimately increase the accuracy of seasonal forecasting. This idea is certainly not new and dates back to Lorenz (1956), who highlighted the links between the so-called dynamical and statistical weather forecasting. The former is based on a set of equations describing the physics of the atmosphere (more generally of the Earth system), while the latter uses statistical tools to infer the state of the Earth system at a future time, without any knowledge of the physical mechanisms underlying the 
system itself. Due to the impact of initial and boundary conditions that are determined with increasing accuracy, the dynamical approach is preferred, as the behavior of the Earth system is efficiently modeled via a set of governing equations that produce accurate weather forecasts especially in the short (up to three days ahead) and medium range (up to two weeks ahead). However, for extended-range (one month ahead) and seasonal (up to one year ahead) weather prediction, anomalies in the turbulent atmospheric flow are relevant, and their efficient characterization in terms of spatiotemporal modes of variability, associated with global teleconnections as well as regional phenomena of the Earth system, are of paramount importance to enhance long-range predictability.

The most common method adopted within the weather and climate community to identify coherent structures is called empirical orthogonal function (EOF) analysis (Obukhov 1947; Lorenz 1956; Kutzbach 1967)—see also Hannachi et al. (2007) for a comprehensive review of this technique and related extensions, and Navarra and Simoncini (2010) for a practical guide of traditional EOF methods applied to climate data. Standard EOF analysis, also known as proper orthogonal decomposition (POD), principle component analysis (PCA), Karhunen-Loève transform (KLT), or Hotelling transform, is a modal decomposition technique that extracts coherent structures, or patterns, in the form of modes of variability from data. These modes are computed as the eigenvectors of the (spatially weighted) anomaly covariance matrix. The corresponding eigenvalues measure the variance and optimally rank the modes. The Karhunen-Loève procedure was first derived by Schmidt (1907) and the reader is referred to Sirovich and Everson (1992) for a comprehensive historical review.

In the past few decades, traditional and more advanced variants of this technique have been used to study the modes of variability and the predictability of the Earth system. For example, Davis (1976) explores sea level pressure and sea surface temperature anomalies using EOF. Similarly, Weare et al. (1976) and Weare and Newell (1977) use EOF to investigate sea surface temperature of both the Atlantic and Pacific Oceans and Barnett (1978) analyzes the variability of surface air temperature over the Northern Hemisphere.

Weare and Nasstrom (1982) present an extended EOF technique and apply it to a few examples. Legler (1983) presents the EOF of wind vectors in the tropical Pacific region, while Servain and Legler (1986) applies it to the sea surface temperature and wind stress in the tropical Atlantic region. Similarly, Mac Veigh et al. (1987) applies EOF to wind stress curl over the North Atlantic. Gamage and Blumen (1993) present a wavelet- and a Fourier-based EOF to capture localized low-level cold fronts, and compare them against traditional EOF. Kawamura (1994) applies a varimax-rotated empirical orthogonal function analysis to monthly mean sea surface temperature anomalies. Brunet (1994) generalizes the EOF theory in the context of normal modes on unidirectional sheared flows. Deser and Blackmon (1995) apply EOF to investigate the relationship between tropical and North Pacific sea surface temperature variations. Smith et al. (1996) reconstruct historical sea surface temperatures via standard EOF, while Zhang et al. (1997) uses EOF to study ENSO-like interdecadal variability in the period 1900-93. Mo (2000) studies the relationships between low-frequency variability in the Southern Hemisphere and sea surface temperature anomalies using standard EOF. Thompson and Wallace (2000) identify the Northern Hemisphere annular as the leading EOF of the sea level pressure field. Limpasuvan and Hartmann (2000) carry out a similar analysis on both Southern and Northern Hemisphere annular modes. Lintner (2002) uses EOF to study the interannual variability of global $\mathrm{CO}_{2}$. Marshall (2003) focuses on the southern annular mode, while Newman et al. (2003) focusses on ENSOforced variability. Wang and An (2005) introduce a method for detecting season-dependent modes, which they denote as S-EOF. Hawkins and Sutton (2007) study the variability of the Atlantic thermohaline circulation using three-dimensional EOF. Miller and Dean $(2007 a, b)$ study the shoreline variability via EOF, identifying its spatiotemporal characteristics and its relationships to nearshore conditions. Finally, Wang et al. (2015) introduce an EOF variant with emphasis on predictability to study the Asian summer monsoon rainfall.

EOF has also been adopted to better assess humaninduced versus natural variability in the context of climate change (Min et al. 2011), used to reconstruct incomplete datasets (e.g., Alvera-Azcárate et al. 2005), and applied to other research fields (e.g., Matsuo and Forbes 2010). In addition, it has been employed to discover and correct errors in global weather and climate models (e.g., Barnett 1999; Mu et al. 2004; Pritchard and Somerville 2009). Complementary techniques have been also used in the context of Earth system variability and predictability (e.g., Gamage and Blumen 1993; Mak 1995).

In this paper, we present a spectral variant of EOF analysis, which we term spectral empirical orthogonal function (SEOF) analysis, in conformity with standard nomenclature in weather and climate studies. This approach differs from standard EOF methods employed in the previously cited. It can be shown that SEOF modes are identical to optimal response modes of a stochastically forced nonnormal linear dynamical system if the forcing consists of white noise. Therefore, the results 
obtained from SEOF provide a direct physical interpretation from a dynamical system perspective and open many opportunities in the analysis and physical understanding of Earth system model results (e.g., Farrell and Ioannou 1996; Sardeshmukh and Sura 2009).

The spectral version of EOF dates back to the work of Lumley (1970). It takes advantage of temporal homogeneity, which makes it ideally suited for statistically (wide-sense) stationary data. The method is based on an eigenvalue decomposition of the estimated crossspectral density (CSD) matrix of the data. At each frequency, SEOF yields a set of optimally ranked, timeharmonic and orthogonal modes. In the atmospheric sciences, this decomposition was proposed by North (1984) to establish the theoretical connection between EOFs computed from linear stochastic models and normal modes of Hermitian operators. More recently, Chekroun and Kondrashov (2017) devised a closely related approach called data-adaptive harmonic (DAH) decomposition. In contrast to SEOF, the DAH decomposition is computed in the time domain and relies on an eigenvalue decomposition of a large block-Hankel matrix. Each block contains the spatial cross-correlation coefficients of one segment of the data within a given window size, and segments are shifted by one time step at a time. The authors used the approach to derive stochastic models and furthermore introduced the notion of phase spectra to discriminate between deterministic and stochastic dynamics. In Kondrashov et al. (2018), DAH is applied to Arctic sea ice concentration. Also recently but in a different context, Towne et al. (2018) related SEOF to other decomposition techniques and addressed its relation to forced linear non-Hermitian systems. We reproduce an outline the latter relation in section $2 \mathrm{~h}$ for completeness.

At the core of SEOF lies the estimation of the CSD matrix, which can readily be performed by means of standard spectral estimation techniques, such as Welch's method (Welch 1967). As compared to standard EOF analysis, the estimation step adds an additional layer of complexity, and much longer time series are needed to converge the second-order statistics. However, the strength of SEOF lies precisely in the latter: the method allows to educe the statistically relevant and persistent patterns. A streaming algorithm which updates the SEOF decomposition on-thefly as new data becomes available has been proposed recently to overcome the computational challenges associated with the need to converge second-order statistics (Schmidt and Towne 2019).

We demonstrate that SEOF allows for the efficient discovery of spatiotemporal coherent weather and climate patterns and teleconnections, both for univariate and multivariate datasets. At the same time, it allows for physical interpretation in terms of linear, stochastically forced systems. Therefore, it can complement and extend the capabilities of existing tools adopted within the weather and climate community, detecting and providing insights into the physical mechanisms governing specific modes of atmospheric or oceanic variability, and providing an efficient testing strategy of predictions from nonlinear forecast models against linear theory.

This paper is organized as follows. In section 2, we formally introduce SEOF analysis and discuss some of its important properties. In the main results section, section 3, we analyze different ERA Interim and ERA $20 \mathrm{C}$ reanalysis data to identify a range of meteorological patterns and teleconnections. The reader is referred to Table 1 for a comprehensive overview of the variables and climate patterns covered in this work. Finally, we summarize our results in section 4.

\section{Spectral empirical orthogonal function analysis}

In this section, we discuss the continuous formulation of SEOF in section $2 \mathrm{a}$, its implementation for discrete data based on spectral estimation in section $2 b$, significance testing in section $2 \mathrm{c}$, and briefly relate SEOF to other methods frequently used for the analysis of time series of climate data in section $2 \mathrm{~d}$, and other EOF variants in section $2 \mathrm{f}$ as well as to eigenanalysis of unforced and forced linear dynamical systems in section $2 \mathrm{~h}$, closely following the derivation of Towne et al. (2018).

\section{a. Continuous formulation and important properties}

We start by defining the space and the space-time inner products

$$
\langle\mathbf{u}, \mathbf{v}\rangle_{\mathbf{x}}=\int_{S} \mathbf{u}^{*}(r, \theta, \varphi) \mathbf{v}(r, \theta, \varphi) d S_{r}
$$

and

$$
\langle\mathbf{u}, \mathbf{v}\rangle_{\mathbf{x}, t}=\int_{-\infty}^{\infty} \int_{S} \mathbf{u}^{*}(r, \theta, \varphi, t) \mathbf{v}(r, \theta, \varphi, t) d S_{r} d t,
$$

respectively, where we denote by $d S_{r}=r^{2} \sin \theta d \theta d \varphi$ the surface element of a sphere $S$ with radius $r$ over $(\theta, \varphi) \in$ $[-\pi / 2, \pi / 2] \times[-\pi, \pi]$. As a shorthand, we define the spherical coordinate triplet $\mathbf{x}=(r, \theta, \varphi)$ consisting of radius, geographic latitude and geographic longitude, respectively. In the following, we assume that $\{\mathbf{q}(\mathbf{x}, t)\}$ is a zero-mean stochastic process, and that its realizations span a Hilbert space $\mathscr{H}$ with inner product $\langle\cdot, \cdot\rangle_{\mathbf{x}, t}$ and expectation operator $E\{\cdot\}$, here taken to be the 
TABLE 1. Overview of reanalysis and spectral estimation parameters. We use the full span of both ERA-Interim (Dee et al. 2011) (1 Jan 1979-1 Jan 2018), and of ERA-20C (Poli et al. 2016) (1 Jan 1900-19 Jan 2011), respectively. 2MT = 2-m temperature; TCC = total cloud cover; TTR $=$ top thermal radiation, $\mathrm{TP}=$ total precipitation, $\mathrm{SST}=$ sea surface temperature, $\mathrm{MSL}=$ mean sea level pressure, $(U, V)=$ wind velocity components; (U10, V10) =10-m wind velocity components; "level" indicates the pressure level and "sfc" the surface level; "type" differentiates daily analysis ("an") data from monthly means of daily means ("mmdm") data; $\Delta t$ is the temporal resolution; $T$ is the segment length; $n_{\text {freq }}$ and $n_{\text {blk }}$ are the total number of frequency bins and the total number of blocks, respectively. The observed atmospheric patterns are abbreviated as: $\mathrm{MJO}=$ Madden-Julian oscillation, ENSO $=$ El Niño-Southern oscillation, $\mathrm{PDO}=\mathrm{Pacific}$ decadal oscillation, QBO = quasi-biennial oscillation, MEI = multivariate ENSO index (Wolter and Timlin 1993), BEI = bivariate ENSO index. Curly brackets $\{\cdot\}$ indicate that the contained variables from a multivariate index. *The $U$-wind component data for the three-dimensional example is extracted at pressure levels $1,5,10,50,100,150,200,250,350,450,550,650,750,800,850,900,950$, and $1000 \mathrm{hPa}$.

\begin{tabular}{|c|c|c|c|c|c|c|c|c|c|c|}
\hline \multicolumn{5}{|c|}{ Database } & \multicolumn{3}{|c|}{ SEOF } & \multicolumn{3}{|c|}{ Result } \\
\hline ERA & Variables & Level & Type & $\Delta t$ & $T$ & $n_{\text {freq }}$ & $n_{\mathrm{blk}}$ & Pattern or index & Section & Figures \\
\hline $20 \mathrm{C}$ & SST & $\mathrm{sfc}$ & an & 30 days & $12 \mathrm{yr}$ & 74 & 9 & ENSO, PDO & $3 a$ & Fig. 2 \\
\hline $20 \mathrm{C}$ & MSL & sfc & an & 30 days & $12 \mathrm{yr}$ & 74 & 9 & QBO & $3 a$ & Fig. 3 \\
\hline $20 \mathrm{C}$ & $U, V$ & $10 \mathrm{hPa}$ & an & 30 days & $12 \mathrm{yr}$ & 74 & 9 & QBO & $3 a$ & Figs. 4,5 \\
\hline Interim & TTR, TP & sfc & an & $12 \mathrm{~h}$ & $1 \mathrm{yr}$ & 366 & 39 & MJO & $3 a$ & Figs. 6,7 \\
\hline Interim & $\begin{array}{l}\{\mathrm{SST}, 2 \mathrm{MT}, \mathrm{U} 10 \\
\quad \mathrm{V} 10, \mathrm{MSL}, \mathrm{TCC}\}\end{array}$ & $\mathrm{sfc}$ & $\mathrm{mmdm}$ & $720 \mathrm{~h}$ & $5 \mathrm{yr}$ & 31 & 22 & MEI & $3 b$ & Fig. 8 \\
\hline $20 \mathrm{C}$ & $\{\mathrm{SST}, U\}$ & sfc, $300 \mathrm{hPa}$ & an & $720 \mathrm{~h}$ & $12 \mathrm{yr}$ & 74 & 9 & BEI & $3 b$ & Fig. 9 \\
\hline $20 \mathrm{C}$ & $U$ & $*$ & $\mathrm{mmdm}$ & $744 \mathrm{~h}$ & $12 \mathrm{yr}$ & 73 & 9 & QBO & $3 \mathrm{c}$ & Figs. 10,11 \\
\hline
\end{tabular}

ensemble mean. The goal of SEOF is to identify $\phi(\mathbf{x}, t) \in \mathscr{H}$ that maximizes

$$
\lambda=\frac{E\left\{\left|\langle\mathbf{q}(\mathbf{x}, t), \phi(\mathbf{x}, t)\rangle_{\mathbf{x}, t}\right|^{2}\right\}}{\langle\phi(\mathbf{x}, t), \phi(\mathbf{x}, t)\rangle_{\mathbf{x}, t}} .
$$

Using a variational approach, it can be shown that $\phi(\mathbf{x}, t)$ must satisfy the Fredholm eigenvalue problem:

$$
\int_{-\infty}^{\infty} \int_{S} \mathscr{C}\left(\mathbf{x}, \mathbf{x}^{\prime}, t, t^{\prime}\right) \phi\left(\mathbf{x}^{\prime}, t^{\prime}\right) d \mathbf{x}^{\prime} d t=\lambda \phi(\mathbf{x}, t)
$$

where $\mathscr{C}\left(\mathbf{x}, \mathbf{x}^{\prime}, t, t^{\prime}\right)=E\left\{\mathbf{q}(\mathbf{x}, t) \mathbf{q}^{*}\left(\mathbf{x}^{\prime}, t^{\prime}\right)\right\}$ is the two-point space-time correlation tensor. The frequency-space representation:

$$
\int_{S} \mathscr{S}\left(\mathbf{x}, \mathbf{x}^{\prime}, f\right) \boldsymbol{\psi}\left(\mathbf{x}^{\prime}, f\right) d \mathbf{x}^{\prime}=\lambda(f) \boldsymbol{\psi}(\mathbf{x}, f)
$$

of Eq. (4) is found under the assumption that $\mathbf{q}(\mathbf{x}, t)$ is a wide-sense stationary process whose correlation function is invariant under a translation $\tau=t-t^{\prime}$ in time [i.e., $\left.\mathscr{C}\left(\mathbf{x}, \mathbf{x}^{\prime}, t, t^{\prime}\right)=\mathscr{C}\left(\mathbf{x}, \mathbf{x}^{\prime}, \tau\right)\right]$. The Fourier transform

$$
\mathscr{S}\left(\mathbf{x}, \mathbf{x}^{\prime}, f\right)=\int_{-\infty}^{\infty} \mathscr{C}\left(\mathbf{x}, \mathbf{x}^{\prime}, \tau\right) e^{i 2 \pi f \tau} d \tau
$$

of the correlation tensor $\mathscr{C}\left(\mathbf{x}, \mathbf{x}^{\prime}, \tau\right)$ defines the crossspectral density tensor $\mathscr{S}\left(\mathbf{x}, \mathbf{x}^{\prime}, f\right)$. At each frequency, the frequency-space eigenvalue problem in Eq. (5) yields a countably infinite number of SEOF modes $\boldsymbol{\psi}_{i}(\mathbf{x}, f)$ as eigenvectors (principal components) of the cross-spectral density tensor, and the same number of corresponding modal energies $\lambda_{i}(f)$. For any given frequency $f$, the SEOF modes have the following useful properties:

- time harmonic with a single frequency $f$,

- coherent in both space and time,

- optimally represent the space-time flow statistics,

- optimally ranked in terms of variance

$$
\lambda_{i}(f): \lambda_{1}(f) \geq \lambda_{2}(f) \geq \lambda_{3}(f) \geq \cdots \geq 0,
$$

- spatially orthogonal under Eq. (1):

$$
\left\langle\boldsymbol{\psi}_{i}(\mathbf{x}, f), \boldsymbol{\psi}_{j}(\mathbf{x}, f)\right\rangle_{\mathbf{x}}=\delta_{i j},
$$

- space-time orthogonal under Eq. (2):

$$
\left\langle\boldsymbol{\psi}_{i}\left(\mathbf{x}^{\prime}, f\right) e^{i 2 \pi f t}, \boldsymbol{\psi}_{j}\left(\mathbf{x}^{\prime}, f\right) e^{i 2 \pi f t}\right\rangle_{\mathbf{x}, t}=\delta_{i j}, \quad \text { and }
$$

- optimally expand the Fourier modes of the flow realizations as $\hat{\mathbf{q}}(\mathbf{x}, \mathbf{f})=\sum_{i=1}^{\infty} a_{i}(f) \boldsymbol{\psi}_{i}(\mathbf{x}, f)$, where $a_{i}(f)=$ $\left\langle\hat{q}(\mathbf{x}, f), \boldsymbol{\psi}_{i}(\mathbf{x}, f)\right\rangle_{\mathbf{x}}$ are the expansion coefficients.

A large separation between the first eigenvalue and all subsequent modal energies at a given frequency furthermore indicates low-rank behavior (Schmidt et al. 2018) of the underlying dynamics (i.e., the dominance of a particular physical mechanism that is represented by the first SEOF mode). We will show in section 3 that this property is particularly useful for identifying climate and weather patterns from modal variance spectra.

\section{b. Computation from discrete data}

Given a time series $\mathbf{q}_{i}=\mathbf{q}\left(t_{i}\right) \in \mathbb{R}^{n}$ at discrete time instances $t_{1}, t_{2}, \ldots, t_{n_{t}} \in \mathbb{R}$, we first form the data matrix: 


$$
\mathbf{Q}=\left[\mathbf{q}_{1}-\overline{\mathbf{q}}, \mathbf{q}_{2}-\overline{\mathbf{q}}, \ldots, \mathbf{q}_{n_{t}}-\overline{\mathbf{q}}\right] \in \mathbb{R}^{n \times n_{t}},
$$

where $\overline{\mathbf{q}}$ is the long-time mean. To estimate the CSD, we segment the data into $n_{\mathrm{blk}}$ overlapping blocks:

$$
\mathbf{Q}^{(l)}=\left[\mathbf{q}_{1}^{(l)}-\overline{\mathbf{q}}, \mathbf{q}_{2}^{(l)}-\overline{\mathbf{q}}, \ldots, \mathbf{q}_{n_{\mathrm{ftt}}}^{(l)}-\overline{\mathbf{q}}\right] \in \mathbb{R}^{n \times n_{\mathrm{fft}}} .
$$

Each block contains $n_{\mathrm{fft}}$ snapshots and overlaps by $n_{\text {ovlp }}$ snapshots with the next segment. The total length of each segment is denoted by $T$. This approach is referred to as Welch's method. The $j$ th column is then given as $\mathbf{q}_{j}^{(l)}=\mathbf{q}_{j+(l-1)\left(n_{\mathrm{fft}}-n_{\text {ovlp }}\right)}-\overline{\mathbf{q}}$. Under the ergodicity hypothesis, each block is regarded as a statistically independent realization. In the present context, however, we found that overlapping the segments did not improve the results, and therefore let $n_{\text {ovlp }}=0$. Next, we obtain the temporal discrete Fourier transform of each block:

$$
\hat{\mathbf{Q}}^{(l)}=\left[\hat{\mathbf{q}}_{1}^{(l)}, \hat{\mathbf{q}}_{2}^{(l)}, \ldots, \hat{\mathbf{q}}_{n_{\mathrm{fft}}}^{(l)}\right] \in \mathbb{R}^{n \times n_{\mathrm{fft}}},
$$

where we use a Hanning window to minimize spectral leakage. Next, we reorganize the data in terms of frequency by collecting all realizations of the Fourier transform at the $k$ th frequency into a single data matrix:

$$
\hat{\mathbf{Q}}_{k}=\left[\hat{\mathbf{q}}_{k}^{(1)}, \hat{\mathbf{q}}_{k}^{(2)}, \ldots, \hat{\mathbf{q}}_{k}^{\left(n_{\mathrm{blk}}\right)}\right] \in \mathbb{R}^{n \times n_{\mathrm{blk}}} .
$$

The weighting

$$
\mathbf{X}_{k}=\frac{1}{\sqrt{n_{\mathrm{blk}}}} \mathbf{W}^{1 / 2} \hat{\mathbf{Q}}_{k}=\left[\mathbf{x}_{k}^{(1)}, \mathbf{x}_{k}^{(2)}, \ldots, \mathbf{x}_{k}^{\left(n_{\mathrm{blk}}\right)}\right] \in \mathbb{R}^{n \times n_{\mathrm{blk}}},
$$

of this matrix will allow us to rank the SEOF modes in terms of the spatial inner product:

$$
\langle\mathbf{u}, \mathbf{v}\rangle_{\mathbf{x}}=\mathbf{u}^{*} \mathbf{W} \mathbf{v},
$$

as the discrete analog to Eq. (2). The numerical quadrature weights (and possibly other weights associated with the discretized inner product) are absorbed into the positive-definite Hermitian matrix $\mathbf{W} \in \mathbb{R}^{n \times n}$. The product

$$
\mathbf{S}_{k}=\mathbf{X}_{k} \mathbf{X}_{k}^{*} \in \mathbb{R}^{n \times n}
$$

defines the CSD matrix of the $k$ th frequency, and its reduced eigenvalue decomposition

$$
\mathbf{S}_{k}=\mathbf{U}_{k} \Lambda_{k} \mathbf{U}_{k}^{*}
$$

constitutes the SEOF. $\boldsymbol{\Lambda}_{k}=\operatorname{diag}\left(\lambda_{k_{1}}, \lambda_{k_{2}} \ldots, \lambda_{k_{n_{\text {blk }}}}\right) \in$ $\mathbb{R}^{n_{\text {blk }} \times n_{\text {blk }}}$ and $\mathbf{U}_{k}=\left[\mathbf{u}_{k_{1}}, \mathbf{u}_{k_{2}}, \ldots, \mathbf{u}_{k_{n_{\text {blk }}}}\right] \in \mathbb{R}^{n \times n_{\text {blk }}}$ are the diagonal matrix of eigenvalues, or modal energies, and the eigenvector matrix, respectively. Finally, the SEOF modes $\phi$ are computed as

$$
\boldsymbol{\Phi}_{k}=\mathbf{W}^{-1 / 2} \mathbf{U}_{k}=\left[\phi_{k_{1}}, \phi_{k_{2}}, \ldots, \phi_{k_{n_{\mathrm{blk}}}}\right] \in \mathbb{R}^{n \times n_{\mathrm{blk}}},
$$

where the weighting guarantees orthonormality

$$
\boldsymbol{\Phi}_{k}^{*} \mathbf{W} \Phi_{k}=\mathbf{I}
$$

under the inner product in Eq. (12).

In practice, the large CSD matrix in Eq. (14) is never formed and decomposed. Instead, we follow Sirovich (1987) and solve the much smaller eigenvalue problem:

$$
\mathbf{X}_{k}^{*} \mathbf{X}_{k} \mathbf{V}_{k}=\mathbf{V}_{k} \boldsymbol{\Lambda}_{k}
$$

for $\mathbf{X}_{k}^{*} \mathbf{X}_{k} \in \mathbb{R}^{n_{\mathrm{blk}} \times n_{\mathrm{blk}}}$ instead of $\mathbf{X}_{k} \mathbf{X}_{k}^{*} \in \mathbb{R}^{n \times n}$ and obtain the SEOF modes from

$$
\boldsymbol{\Phi}_{k}=\mathbf{W}^{-1 / 2} \mathbf{X}_{k} \mathbf{V}_{k} \boldsymbol{\Lambda}_{k}^{-1 / 2}
$$

Owing to the symmetry of the Fourier transform for real signals, such as the ones under investigation in this work, it is sufficient to consider positive frequencies. The total number of frequency bins in this case is given as

$$
n_{\text {freq }}=\left\lceil\frac{n_{\text {fft }}}{2}\right\rceil+1 \text {. }
$$

\section{c. Significance levels}

From the hypothesis that the time series $\mathbf{q}(t)$, and therefore also its Fourier transform $\hat{\mathbf{q}}(f)$, are normally distributed, it follows that the coefficients $a_{k_{j}}^{(i)}$ that expand the $i$ th Fourier realization at the $k$ th frequency as

$$
\hat{\mathbf{q}}_{k}^{(i)}=\sum_{j=1}^{n_{\mathrm{blk}}} a_{k_{j}}^{(i)} \phi_{k_{j}}
$$

are also normally distributed. Leveraging the orthogonality of the eigenvectors at each frequency, the expansion coefficients can readily be obtained from the projection of the Fourier realizations onto the SEOF modes,

$$
a_{k_{j}}^{(i)}=\left\langle\hat{\mathbf{q}}_{k}^{(i)}, \phi_{k_{j}}\right\rangle_{\mathbf{x}} .
$$

By construction, the expansion coefficients are uncorrelated and their variance is therefore given by the eigenvalues of the CSD matrix, that is,

$$
E\left\{a_{k_{i}} a_{k_{j}}^{*}\right\}=\lambda_{k_{j}} \delta_{i j}=\frac{1}{n_{\mathrm{blk}}} \sum_{i=1}^{n_{\mathrm{blk}}}\left|a_{k_{j}}^{(i)}\right|^{2}
$$


Equation (18) shows that the SEOF eigenvalues are estimated as the sum of squares of the expansions coefficients. Now, since the coefficients $a_{k_{j}}^{(i)}$ are uncorrelated and normally distributed, we expect $\left|a_{k_{j}}^{(i)}\right|^{2}$ to be chisquare distributed. Analogous to the case of averaging overlapping periodograms (Welch 1967; Manolakis et al. 2005), the $(1-\alpha) \times 100 \%$ confidence interval that either contains or does not contain the true eigenvalue $\lambda_{k_{j}}$ is hence given by

$$
\left[\frac{2 n_{\mathrm{blk}} \lambda_{k_{j}}}{\chi_{2 n_{\mathrm{blk}}}^{2}(1-\alpha / 2)}, \frac{2 n_{\mathrm{blk}} \lambda_{k_{j}}}{\chi_{2 n_{\mathrm{blk}}}^{2}(\alpha / 2)}\right],
$$

where $\chi_{2 n_{\mathrm{blk}}}^{2}$ is the chi-square distribution with $2 n_{\mathrm{blk}}$ degrees of freedom.

\section{d. Relations to the discrete Fourier transform, power spectra, and singular spectrum analysis (SSA)}

In the following, we briefly address a few popular methods that are frequently used to analyze time series of climate data, and relate them to SEOF. For a comprehensive review with an emphasis on climate data, the reader is referred to Ghil et al. (2002). The most straightforward way to analyze the frequency content of a time series is to obtain an estimate of a power spectrum, defined as the mean squared absolute value of the signal as a function of frequency, by applying the discrete Fourier transform directly to the data. This, however, leads to an approximation error that is of the same order as the variance of the signal. For this reason, spectral estimation techniques such as Welch's method or multitaper methods are used to reduce the variance of the estimate in practice. Similarly, we apply Welch's method to the realizations of the Fourier transform in Eqs. (8)-(11) above. The SEOF spectra, therefore, inherit some of the properties of power spectra. The trade-offs involved in choosing appropriate spectral estimation parameters to avoid aliasing and spectral leakage can be found in standard textbooks on signal processing like Manolakis et al. (2005).

\section{e. Relation with singular spectrum analysis (SSA)}

While spectral analysis methods decompose the data into purely oscillatory patterns, the widely popular singular spectrum analysis (SSA) is data adaptive and allows for the separation of oscillatory patterns, trends and noise from univariate time series (Ghil et al. 2002). SSA computes temporal EOF modes as the eigenvectors of the lag-covariance matrix. Closely related to the present work is the extension of SSA to multidimensional data proposed by Plaut and Vautard (1994), which leads to a space-time formulation referred to as multichannel singular spectrum analysis (M-SSA). For a recent application of M-SSA to Simple Ocean Data Analysis
(SODA) reanalysis data with many technical details on the method, the reader is referred to Groth et al. (2017). If M-SSA were conducted in the frequency domain, it would correspond to SEOF with $n_{\text {ovlp }}=n_{\mathrm{fft}}-1$.

\section{f. Connections with other EOF variants}

An overview of different flavors of EOF used in meteorology studies can be found in Hannachi et al. (2007). Of all methods described in the latter review, frequency domain EOF analysis (FDEOF) is the approach that is the most similar to SEOF. Just like SEOF, FDEOF is based on an eigenvalue decomposition of the CSD matrix. However, unlike SEOF, FDEOF does not use a spectral estimate of the CSD. Instead, the cross-spectral matrix for a given frequency is written in terms of the time-lagged covariance matrix, and the average CSD over some frequency band is analyzed. According to Hannachi et al. (2007), FDEOF has been abandoned and replaced by complex Hilbert EOF analysis (HEOF) due to certain shortcomings related to the frequency band averaging procedure. HEOF, however, is a timedomain technique and cannot directly be used to educe patterns that oscillate at a single frequency.

\section{g. Connection with empirical normal modes (ENM)}

A criticism (see, e.g., Monahan et al. 2009) of EOF analysis is that individual EOF modes do not, for general nonnormal operators for which $\mathbf{A}^{\dagger} \mathbf{A} \neq \mathbf{A A}^{\dagger}$, correspond to dynamical modes [i.e., the eigenvectors of the linear dynamical operator $\mathbf{A}=\mathbf{A}\left(\mathbf{q}_{0}\right)$ that governs the evolution]:

$$
\frac{\partial \mathbf{q}}{\partial t}=\mathbf{A q}
$$

of the perturbation quantity $\mathbf{q}$ about a base state $\mathbf{q}_{0}$. This apparent drawback can partially be mitigated by enforcing pseudomomentum or pseudoenergy orthogonality in terms of conservation laws (Held 1985). The empirical normal mode (ENM) analysis technique introduced by Brunet (1994) leverages this idea to compute dynamical modes of variability that are directly linked to characteristic physical properties like intrinsic frequency or a phase speed. Using conserved pseudomomentum and pseudoenergy wave activities, Brunet (1994) demonstrated the correspondence between EOFs and the normal modes of the primitive atmospheric equation for sheared flows. ENM analysis has been successfully applied to reanalysis data, for example the multilayer NCEP winter reanalyses (Zadra et al. 2002). As for standard EOF, a space-time variant of ENM (called ST-ENM) is readily obtained by decomposing the space and time-lag covariance matrix (Martinez et al. 2011). When applied in the frequency domain, 
this approach would correspond to pseudoenergy or pseudomomentum-based SEOF.

\section{h. Connection with optimal excitation responses}

In a large body of work, prominently represented by their seminal contribution on generalized stability theory, Farrell and Ioannou (1996) argue that atmospheric dynamics are best modeled by a stochastically forced nonnormal linear dynamical system:

$$
\frac{\partial \mathbf{q}}{\partial t}=\mathbf{A q}+\mathbf{f}(t),
$$

where $\mathbf{f}=\mathbf{f}(t)$ is the external stochastic forcing which mimics the background turbulence or unresolved physical scales. Using the Fourier transform pair:

$$
\begin{gathered}
\mathbf{q}(t)=\int_{-\infty}^{\infty} \hat{\mathbf{q}}(\omega) e^{i \omega t} d \omega \\
\hat{\mathbf{q}}(\omega)=\frac{1}{2 \pi} \int_{-\infty}^{\infty} \mathbf{q}(t) e^{-i \omega t} d t
\end{gathered}
$$

a direct relation:

$$
\hat{\mathbf{q}}(\omega)=(i \omega \mathbf{I}-\mathbf{A})^{-1} \hat{\mathbf{f}}(\omega)=\mathbf{R} \hat{\mathbf{f}}(\omega)
$$

between responses $\hat{\mathbf{q}}(\omega)$ and forcings $\hat{\mathbf{f}}(\omega)$ at frequency $\omega$ via the transfer function $\mathbf{R}=\mathbf{R}(\omega)=(i \omega \mathbf{l}-\mathbf{A})^{-1}$, sometimes referred to as the resolvent operator, is found. The singular value decomposition (SVD) $\mathbf{R}=\mathbf{U} \Sigma \mathbf{V}^{*}$ of the discretized resolvent operator yields responses (columns of $\mathbf{U}$ ) to optimal excitation distributions, or forcings, (columns of $\mathbf{V}$ ) that describe the continuously forced linear system in Eq. (21) in a way similar to the eigenanalysis of $\mathbf{A}$, which characterizes the asymptotic behavior of the unforced linear system in Eq. (20) as $t \rightarrow \infty$. The optimal forcings and responses are optimally ranked in terms of the energetic gain given by the singular values $\operatorname{diag}(\Sigma)$. An interesting correspondence between SEOF modes and the optimal responses can be derived as follows (Towne et al. 2018). We start by defining the CSDs of the response $\mathbf{S}_{\hat{\mathbf{q}} \hat{\mathbf{q}}}=E\left\{\hat{\mathbf{q}}(\omega) \hat{\mathbf{q}}^{*}(\omega)\right\}$ and the forcing $\mathbf{S}_{\hat{\mathbf{f}} \hat{\mathbf{f}}}=E\left\{\hat{\mathbf{f}}(\omega) \hat{\mathbf{f}}^{*}(\omega)\right\}$, respectively. Next, we make use of Eq. (24) to express the response CSD tensor in terms of the resolvent operator and the forcing CSD as

$$
\begin{aligned}
\mathbf{S}_{\hat{\mathbf{q}} \hat{\mathbf{q}}} & =E\left\{\hat{\mathbf{q}}(\omega) \hat{\mathbf{q}}^{*}(\omega)\right\}=E\left\{\mathbf{R} \hat{\mathbf{f}}(\omega) \hat{\mathbf{f}}^{*}(\omega) \mathbf{R}^{*}\right\} \\
& =\mathbf{R} E\left\{\hat{\mathbf{f}}(\omega) \hat{\mathbf{f}}^{*}(\omega)\right\} \mathbf{R}^{*}=\mathbf{R} \mathbf{S}_{\hat{\mathbf{f f}}} \mathbf{R}^{*}
\end{aligned}
$$

Under the common modeling assumption that the forcing is uncorrelated in both time and space and of unit amplitude everywhere (see, e.g., Farrell and Ioannou 1996; Towne et al. 2018), the forcing CSD reduces to $\mathbf{S}_{\hat{\mathbf{f}} \hat{\mathbf{f}}}=\mathbf{I}$, and we can express the response CSD in terms of the SVD of the resolvent operator as

$$
\mathbf{S}_{\hat{\mathbf{q}} \hat{\mathbf{q}}}=\mathbf{R S}_{\hat{\mathbf{f f}}} \mathbf{R}^{*}=\mathbf{R} \mathbf{R}^{*}=\mathbf{U} \Sigma \mathbf{V}^{*} \mathbf{V} \Sigma \mathbf{U}^{*}=\mathbf{U} \Sigma^{2} \mathbf{U}^{*} .
$$

From comparing this expression with Eq. (14) above, it is concluded that SEOF response modes are identical to optimal response modes of the forced linear system in Eq. (21) if the forcing $\mathbf{f}(t)$ is white in space and time.

\section{Results}

We apply SEOF to extract statistical modes of variability from two climate reanalysis datasets, ERA-Interim (Dee et al. 2011) and ERA-20C (Poli et al. 2016). On one hand, we leverage the high temporal resolution of the ERA-Interim dataset to educe patterns that evolve on time scales of a few months. The large temporal span of ERA-20C, on the other hand, allows us to converge the statistics of patterns with periods of many years. Table 1 summarizes the databases and SEOF parameters used to educe the different patterns. In the rest of this section, we present the results for each mode of variability (also referred to as oscillation) reported in the table.

The two main outcomes of a SEOF analysis are the SEOF modes $\phi_{i}$ as defined in Eq. (15), which represent that spatial pattern of the oscillation, and their corresponging modal energies $\lambda_{j}$, as defined in Eq. (14). For brevity, we use a single subscript $j$ for the mode index and omit the frequency index $k$. The modal energies can be represented in the form of spectra by plotting one curve for each mode number over frequency. It is important to note that the representation by a curve leads to an intuitive representation of the modal energies, but does not imply continuity of the mode pattern when the frequency is varied. For example, the first mode of two neighboring frequencies may represent two different physical phenomena. An example of this representation is shown in Fig. 1 (left). The distinct peaks at 365 days, 183 days and so on represent annual and seasonal cycles that are, however, not of primary interest for this analysis. Many of the climate patterns that are of interest can better be identified by their low-rank behavior. To this end, we define the normalized modal variance difference:

$$
\Delta \lambda=\frac{\lambda_{1}-\lambda_{2}}{\sum_{j=1}^{n_{\text {blk }}} \lambda_{j}}
$$

as the normalized difference in variance, between the first and second mode. The corresponding plot is shown in Fig. 1 (right). Besides the annual cycle, the spectrum exhibits a distinct peak centered around a period of 

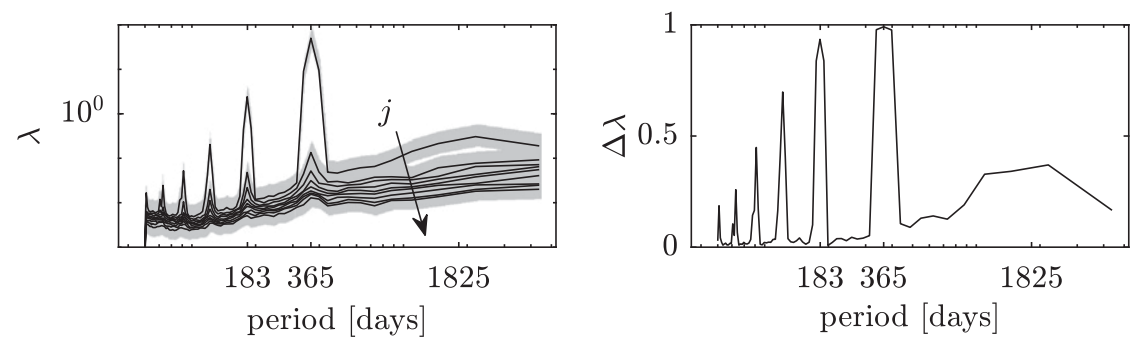

FIG. 1. Representation of the SEOF modal variance in the form of spectra. (left) Spectra for all $n_{\mathrm{blk}}$ modes with $j$ as the mode index. For example, the top line with $j=1$ describes the variance of the leading mode that captures the largest fraction of the total variance at any given frequency. The gray shaded area indicates the $95 \%$ confidence intervals for the spectra. (right) Normalized difference between the first and second modal energies as defined by Eq. (27). The SEOF spectrum of the sea surface temperature (SST) according to the parameters used to identify the ENSO and PDO in Table 1, line 1, is shown as an example.

approximately 5 years. We will show in the next section that this peak is the footprint of the ENSO and PDO. In the reminder of the paper, starting with Fig. 2, we use a comprehensive plot showing the real part of the leading SEOF mode at the frequency of interest (top left), the $\Delta \lambda$ spectrum (bottom right), and Hovmöller diagrams in the longitudinal and latitudinal directions at the location of maximum absolute value of the mode over one period in time (bottom left and top right, respectively). The frequency of interest is indicated by the magenta line in the spectrum and the location of maximum absolute value of the mode as the intersection of the magenta lines in the contour plot of the modal structure. In this paper, we focus our attention to the leading SEOF mode. The baseline approach shown in the following section $3 a$ is to use surface data of a single variable to compute univariate two-dimensional SEOF modes. In addition, we demonstrate how the method can be extended to compute multivariate modes (i.e., modes consisting of multiple variables that form an index), and three-dimensional modes that represent structures that are perfectly coherent not only at the surface, but also at different height bands in sections $3 \mathrm{~b}$ and $3 \mathrm{c}$, respectively.
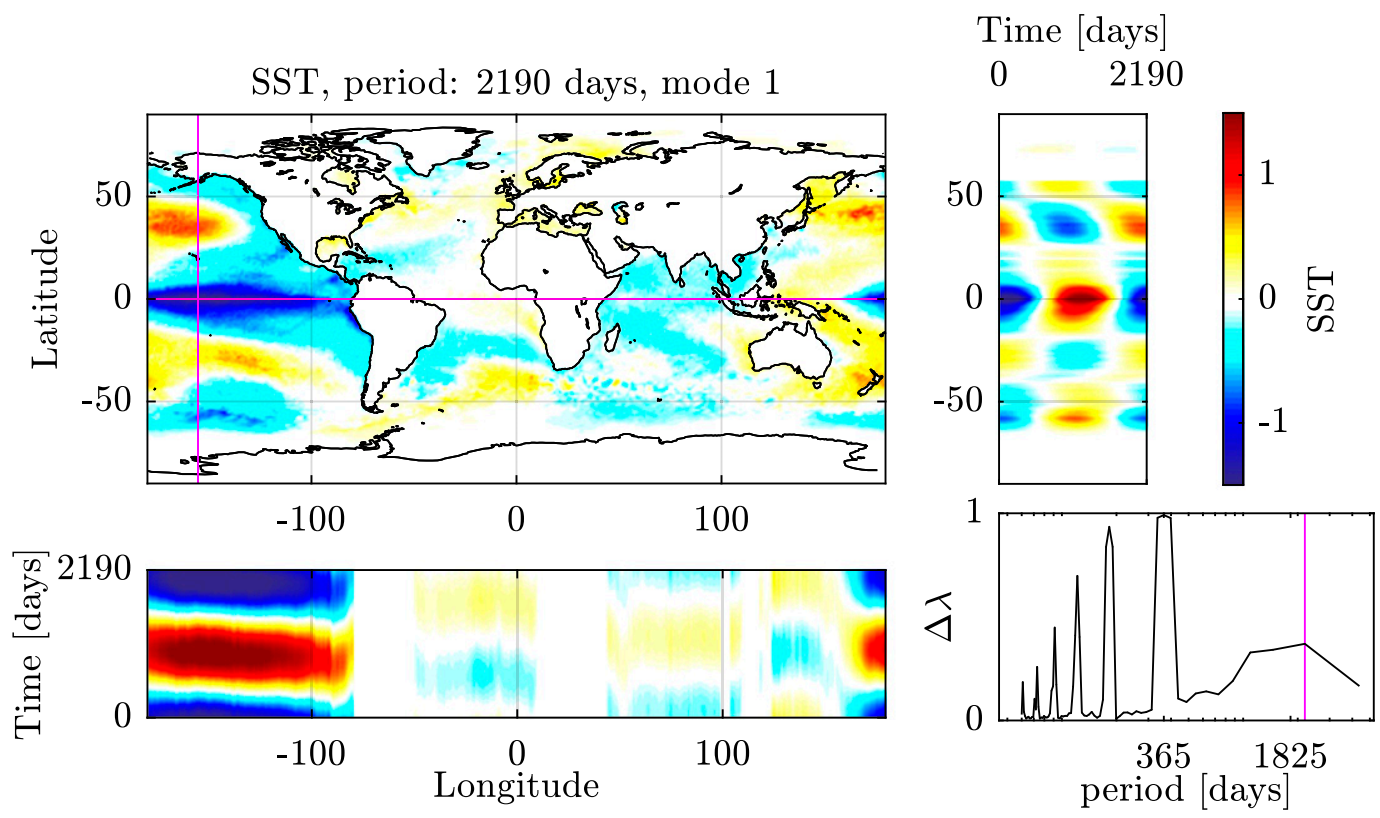

FIG. 2. SEOF analysis for the sea surface temperature (SST): (top left) real part of the leading SEOF mode with period 2190 days; (bottom right) spectrum of the normalized modal variance difference $\Delta \lambda$; Hovmöller diagrams in the (bottom left) longitudinal and (top right) latitudinal directions at the location of maximum absolute value of the mode over one period. The frequency of interest is indicated by the magenta line in the spectrum and the location of the maximum absolute value of the mode as the intersection of the magenta lines in the contour plot of the modal structure. 

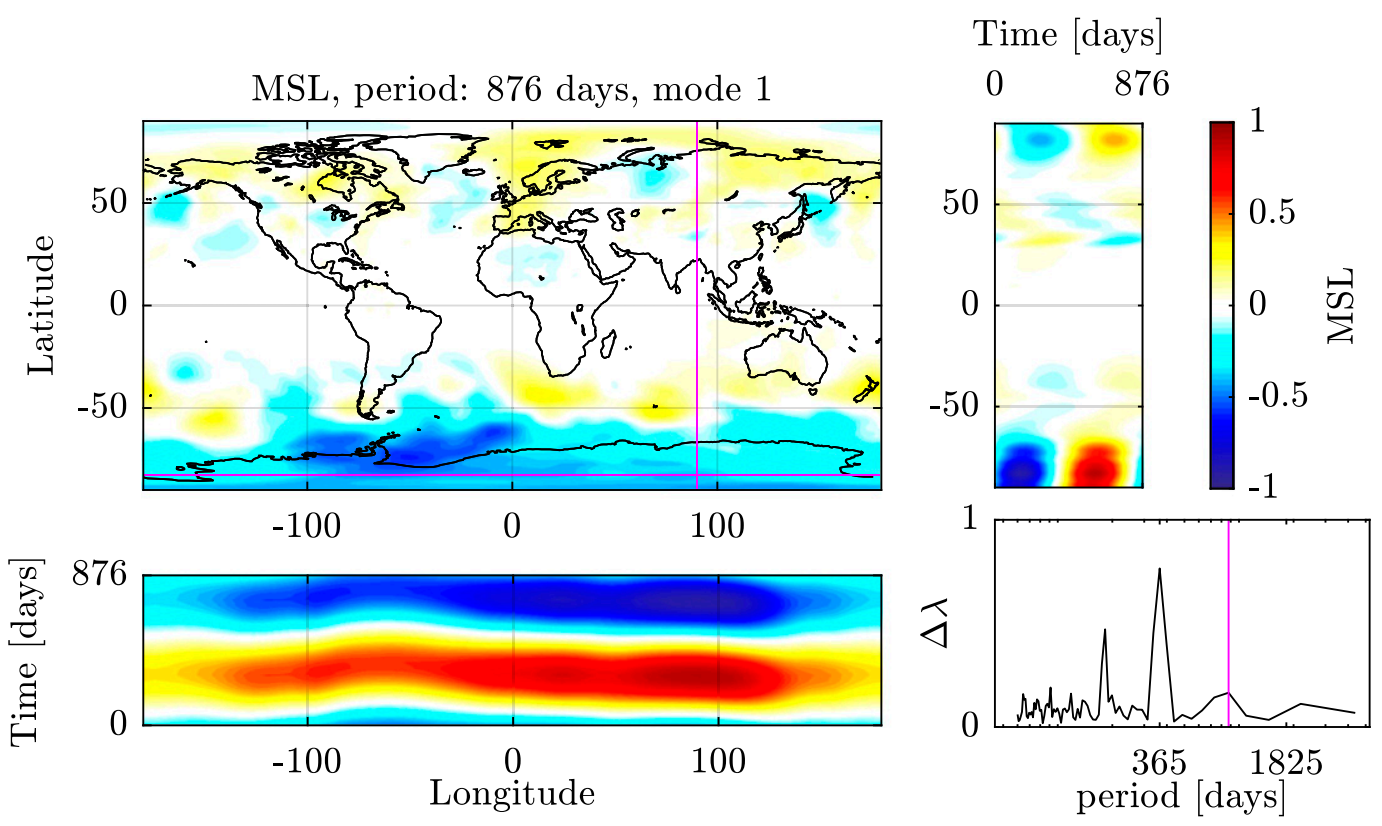

FIG. 3. As in Fig. 2, but for the mean sea level pressure (MSL) and period 876 days, identifying the quasi-biennial oscillation.

\section{a. Univariate two-dimensional data}

1) El NiÑO-SOUTHERN OSCILLATION AND LINKS TO PACIFIC DECADAL OSCILLATION (PDO) PATTERNS

\section{2-7-yr (ENSO) and 8-12-yr (PDO) periodicity}

The El Niño-Southern Oscillation analyzed in Fig. 3 involves coupled atmospheric-ocean processes, and represents the strongest year-to-year fluctuation of the global climate system, affecting ecosystems as well as human activities. While it has been widely studied throughout the past few decades, its complexity makes it difficult to reach a full understanding of both large- and small-scale effects of this phenomenon, as well as of its implications on other modes of variability (Timmermann et al. 2018). ENSO is characterized by the periodic change in winds and sea surface temperature (SST) in the tropical eastern Pacific Ocean, coupled with periodic air surface pressure variation in the tropical western Pacific Ocean. The individual ENSO periods last several months each (typically occurring every few years) and their effects vary in intensity and complexity (see, e.g., Trenberth 1997).

ENSO-like, the Pacific decadal oscillation is a coupled ocean-atmosphere process, that is however, most visible in the North Pacific region with alternating patterns of SST [see, e.g., Mantua and Hare (2002) for further details].

Interestingly, the SEOF analysis identifies and links both, the anomalous patterns in the North Pacific reminiscent of the PDO and the equatorial SST. In Fig. 2, the ENSO and the PDO patterns are identified in the SST. In the SEOF mode depicted in Fig. 2, the occurrence of these two patterns is evident. In the normalized power spectrum, the ENSO pattern materializes in the form of a broad crest that is centered about a 5-yr-period peak. The spread around the peak is in accordance with the typical ENSO variability.

The interplay between the ENSO and PDO has been the focus of several recent studies (e.g., Wang et al. 2014), and has strong implications in terms of teleconnections and global circulation patterns, among others.

\section{2) QuASI-BIENNIAL OSCILLATION (QBO)}

\section{8-29-month periodicity}

The QBO exhibits quasi-periodic reversals of the zonalmean zonal winds in the equatorial stratosphere between easterlies and westerlies with a distinct apparent downward phase propagation of the alternating wind regimes [see, e.g., Baldwin et al. (2001) for further details]. This mode of variability has important implications in terms of teleconnections with several weather patterns in the Northern Hemisphere, including averaged zonal winds, mean sea level pressure and tropical precipitation (Gray et al. 2018).

It can be seen that the QBO is most unambiguously identified in terms of the $10-\mathrm{hPa} U$ component of the wind velocity shown in Fig. 4. The corresponding spatial pattern is a clear latitudinal band in $-20^{\circ} \lesssim \phi \lesssim 20^{\circ}$. The 10-hPa $V$ wind component and the mean sea level pressure (MSL) shown in Figs. 3 and 5, respectively, exhibit a significantly less distinct peak in the spectra around the typical QBO period. A more comprehensive SEOF analysis can uncover teleconnections as well as 

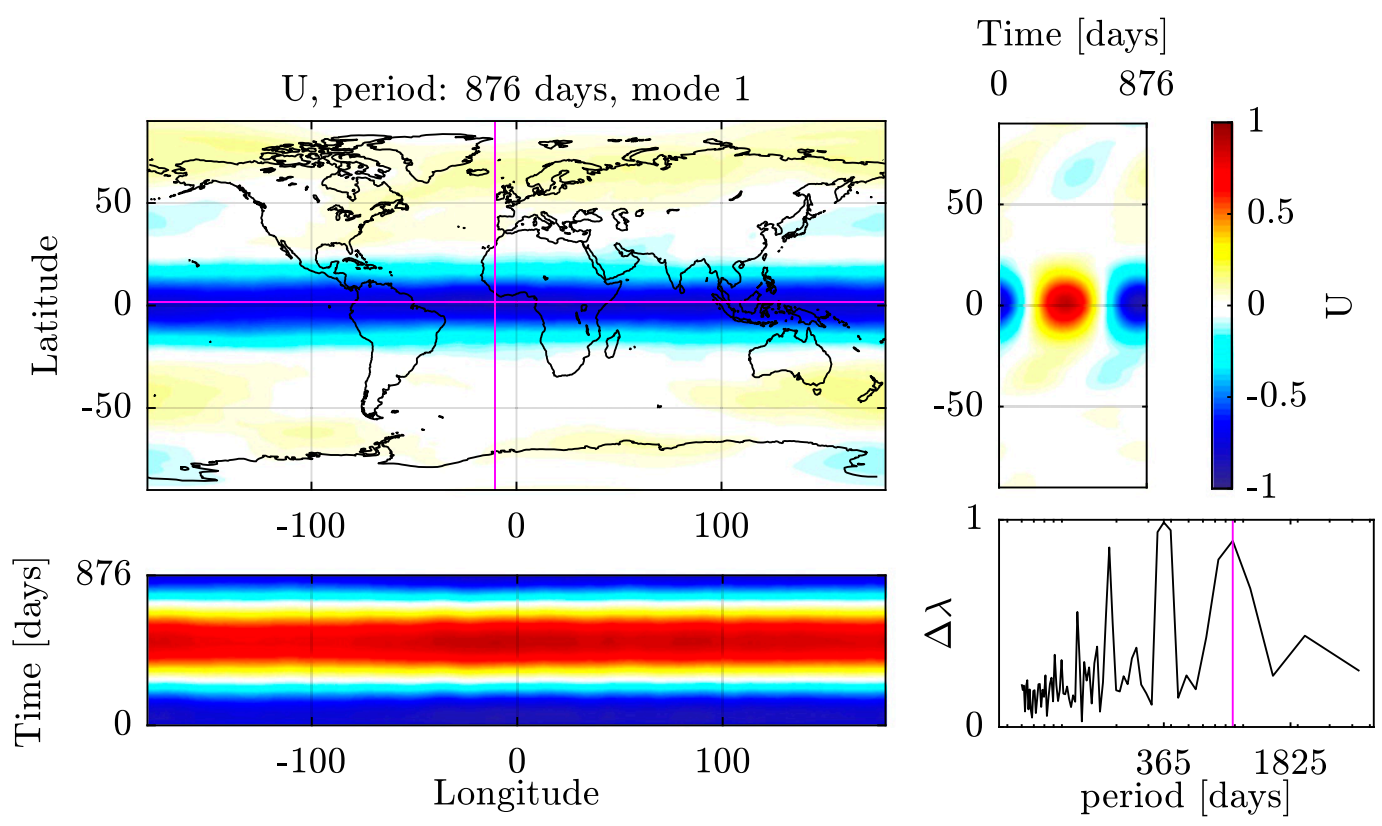

FIG. 4. As in Fig. 2, but for the 10-hPa $U$ velocity $(U)$ and period 876 days, identifying the quasi-biennial oscillation.

the interplay of this oscillation with other coherent structures that have possibly remained unidentified. This, however, is beyond the scope of this paper.

\section{3) MAdDEn-Julian Oscillation (MJO)}

\section{0-90-day periodicity}

The MJO is the largest component of intraseasonal variability in the tropical atmosphere. It is not strictly an oscillation as its period varies and its appearance is episodic Hartmann and Hendon (2007). It is characterized by a large-scale coupling between atmospheric circulation and tropical deep convection that manifests itself in an eastward propagating planetary-scale (time-mean) flow anomaly traveling at $4-8 \mathrm{~m} \mathrm{~s}^{-1}$ through the atmosphere above the warm parts of the Indian and Pacific Oceans. Its signature can be identified by anomalous rainfall and significantly increased convective activity
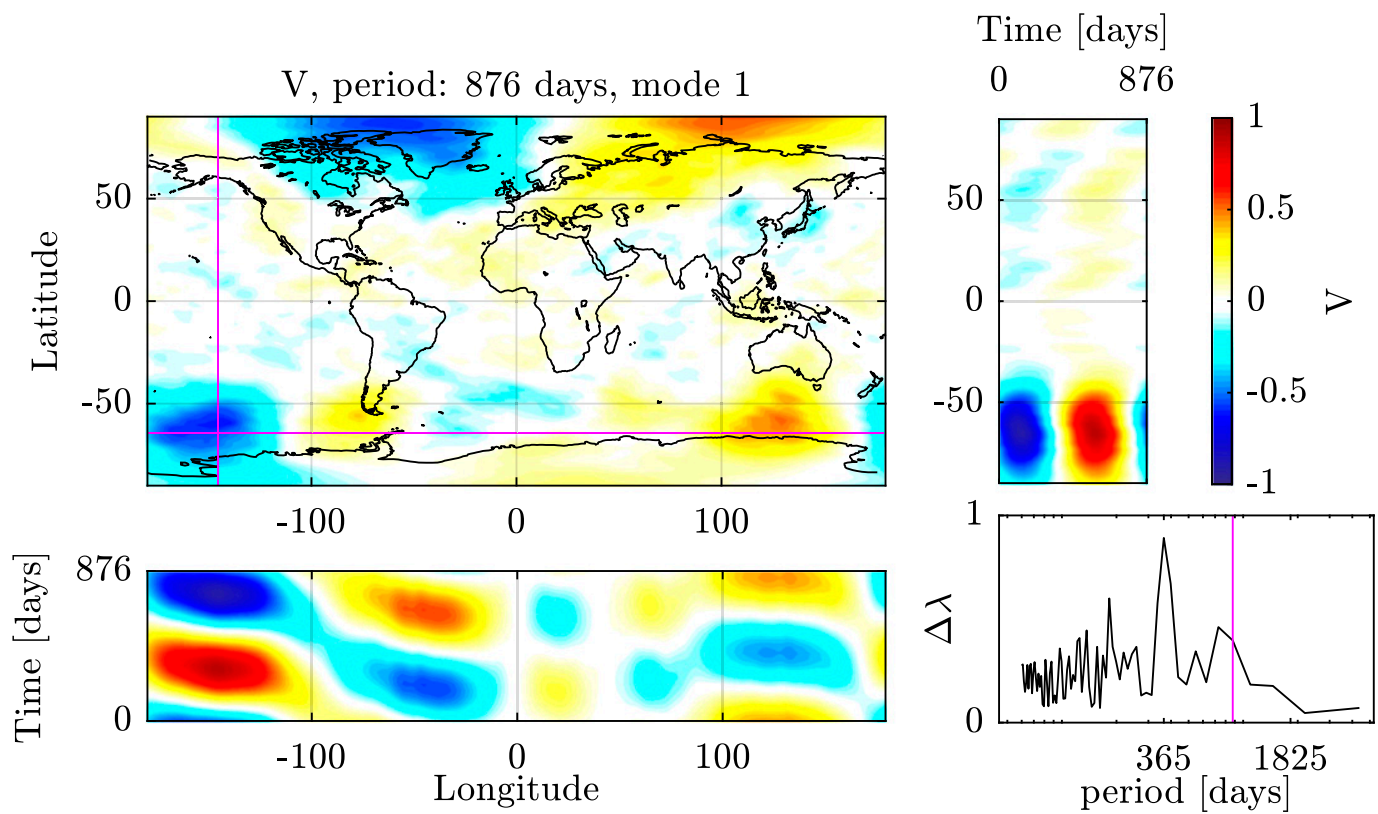

FIG. 5. As in Fig. 2, but for the 10-hPa $V$ velocity $(V)$ and period 876 days, identifying the quasi-biennial oscillation. 


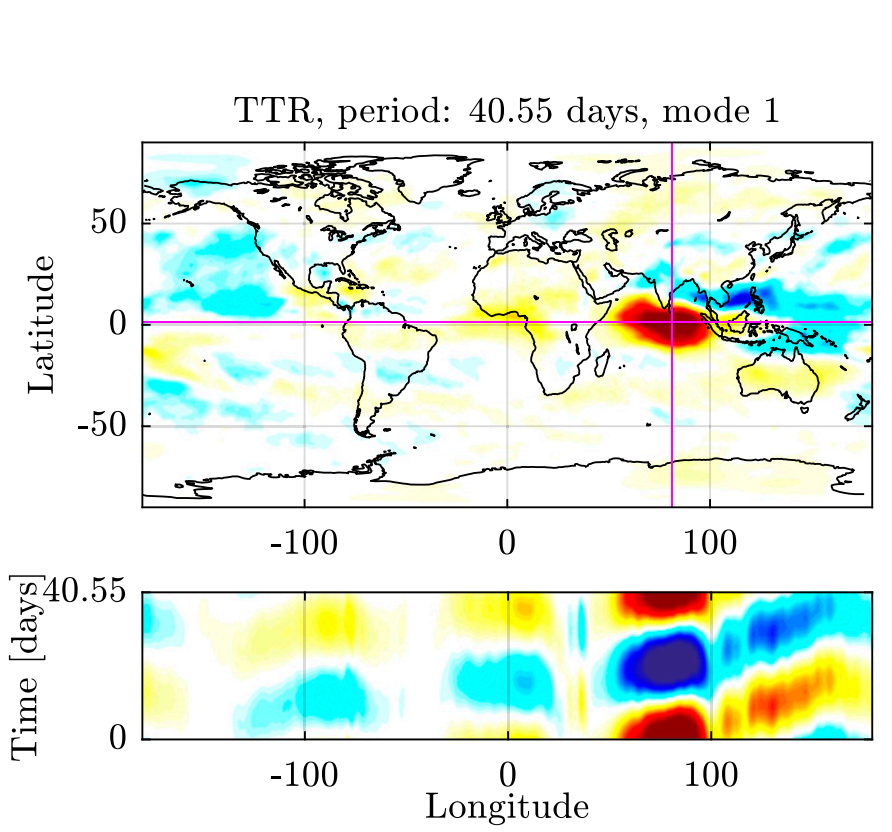

Time [days]

$0 \quad 40.55$
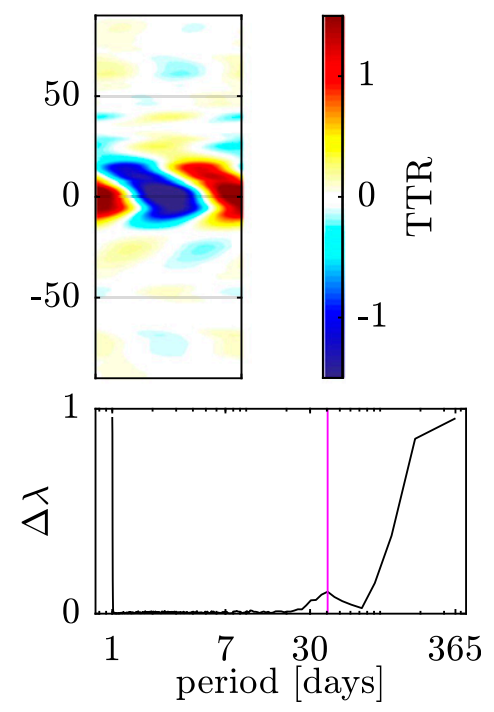

FIG. 6. As in Fig. 2, but for the top thermal radiation (TTR) and period 40.55 days, identifying the Madden-Julian oscillation.

traced by its outgoing longwave radiation (OLR), and it has been associated with extreme weather events at midlatitudes [see, e.g., Zhang (2005) for further details].

In Figs. 6 and 7, we identify the MJO in the top thermal radiation (TTR) and the total precipitation (TP), respectively. For both quantities, we observe (besides the dominant diurnal and annular peaks) distinct peaks in the spectra, and a clear separation between the variance of the leading SEOF mode and all other modes. The peaks are centered at about 43 days and their width corresponds approximately to the anticipated 30-90-day periodicity.

\section{b. Multivariate two-dimensional data}

As an example of a multivariate index, we adapt the multivariate ENSO index (Wolter and Timlin 1993) (MEI) for SEOF. Multivariate indices are used to understand the

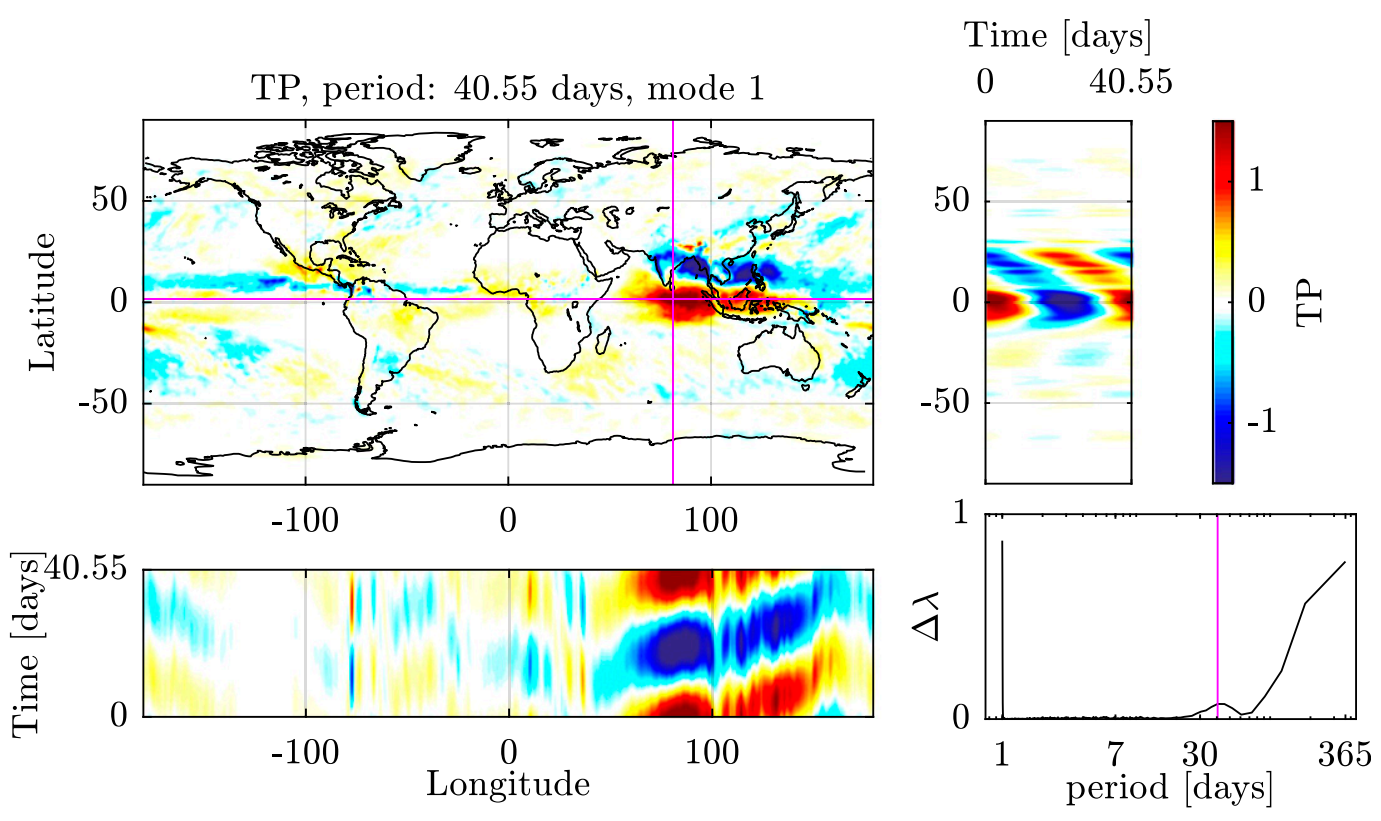

FIG. 7. As in Fig. 2, but for the total precipitation (TP) and period 45.6 days identifying the Madden-Julian oscillation. 


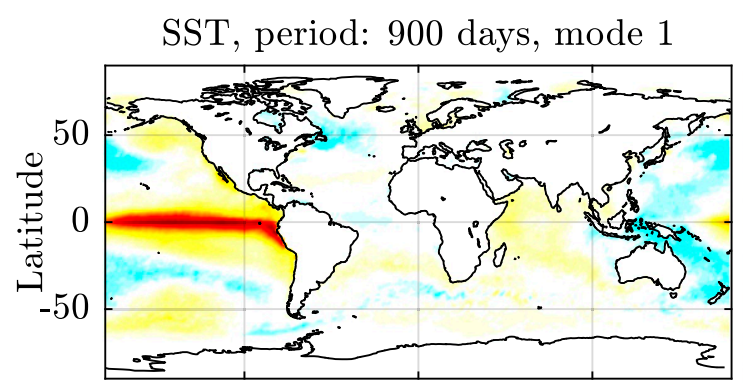

U10, period: 900 days, mode 1

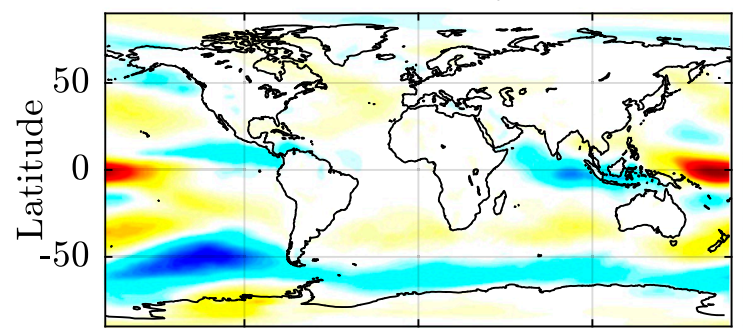

MSL, period: 900 days, mode 1

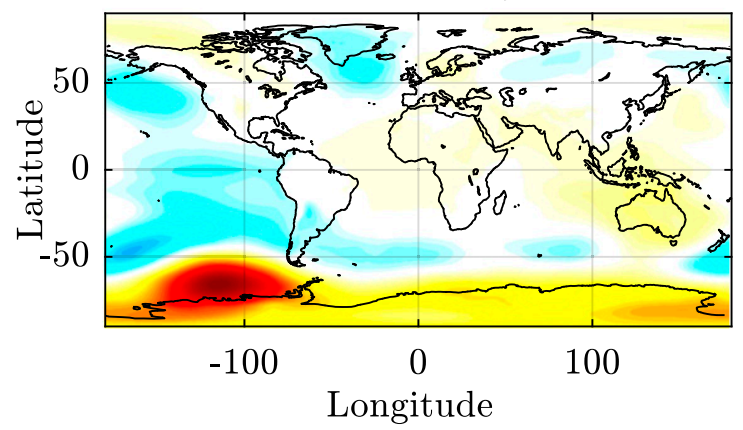

T2M, period: 900 days, mode 1

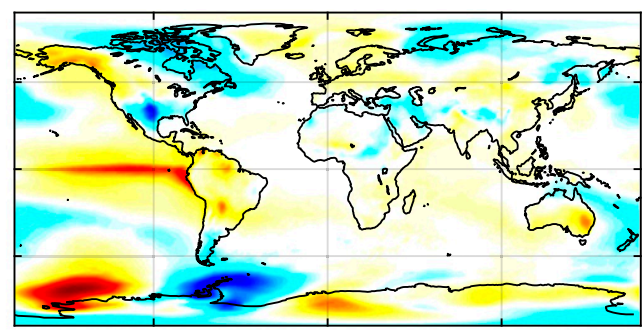

V10, period: 900 days, mode 1

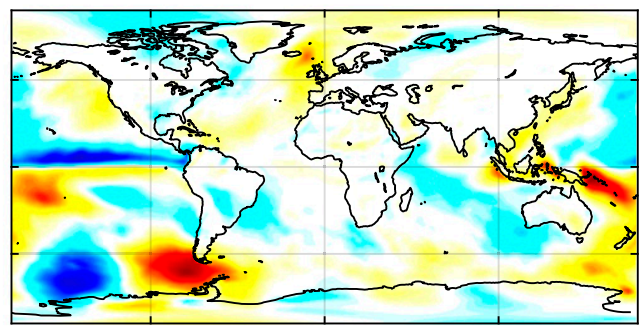

TCC, period: 900 days, mode 1

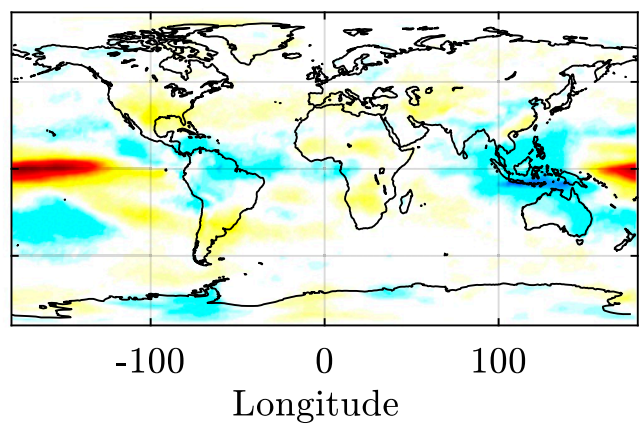

FIG. 8. Multivariate ENSO index based on the set of variables defined by Wolter and Timlin (1993). The same visualization as in Fig. 2 is used for each individual field of index.

interplay of different quantities by slaving them together into a single index that then describes coherent patterns correlated across different quantities. The MEI consists of six variables: mean sea level pressure (MSL), zonal and meridional components of the surface wind (U10, V10), sea surface temperature (SST), 2-m temperature (T2M), and total cloud cover (TCC). As in the original work by Wolter and Timlin (1993), we equalize the importance of the different quantities by normalizing each field by its total variance. We do not, however, consider sliding bimonthly seasons as in the original work, but apply SEOF in accordance with the spectral estimation parameters shown in Table 1. The leading SEOF mode over all six fields for a period of 900 days is reported in Fig. 8. Besides the typical signature of the ENSO, most prominently seen in SST, T2M, V10, and TCC, a vortex pattern over West Antarctica, between the Antarctic Peninsula and the Ross Sea, is observed in T2M, U10, V10, and MSL. A direct coupling between the two structures is apparent in T2M and the 10-m wind components. A similar link was previously reported by Clem and Fogt (2013) and tied with the strength and phase of the southern annular mode, thereby linking ENSO to the Amundsen Antarctica ice-shelf height variability (Paolo et al. 2018).

The MEI example above demonstrates how SEOF can be used to investigate the interplay of different fields by combining them into an index. Naturally, the opposite also holds true (i.e., SEOF can be used to show statistical independence of different phenomena). In Fig. 9, we define a bivariate index comprising SST and high-latitude wind $(300 \mathrm{hPa})$ to inspect an apparent connection between ENSO and Southern Hemisphere high-latitude zonal-mean winds. As shown by Byrne et al. (2017), this connection disappears once the signature of the stratospheric vortex breakdown is removed. Consistent with their results, no dominant zonal wind structure is observed in Fig. 9. Instead, we observe a similar pattern 


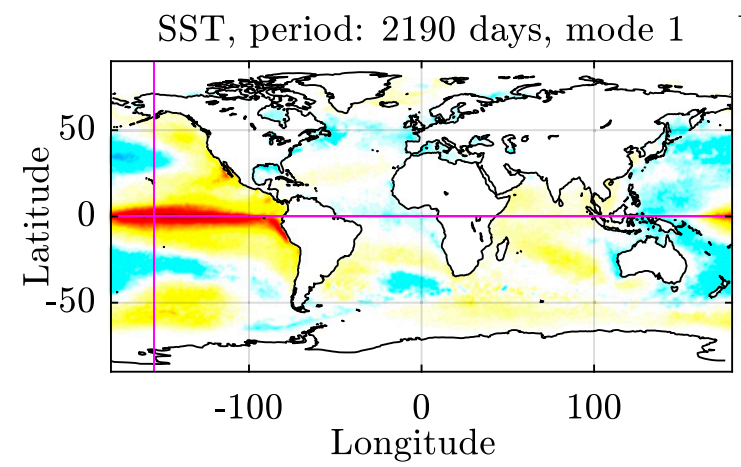

$\mathrm{U} @ 300$ hPa, period: 2190 days, mode 1

FIG. 9. Bivariate index defined to inspect the supposed correlation between ENSO and the zonal-mean zonal wind in the lower stratosphere of the Southern Hemisphere. The same visualization as in Fig. 2 is used for each individual field of index.

to the U10 component in the MEI index over the Pacific, as depicted in Fig. 8. In this example, the assumption of stationarity helps to separate coherent motion from rare nonstationary events, like the breakup of the stratospheric vortex. On the other hand, seasonal variations are almost sufficiently periodic. Consequently, they are resolved at their corresponding period.

\section{c. Three-dimensional data}

In this section, we demonstrate how SEOF can directly be applied to three-dimensional data. As an example, we educe the QBO from monthly means of daily means data of the $U$-wind component from ERA $20 \mathrm{C}$ data at 18 pressure levels. Figure 10 shows the familiar two-dimensional visualization of the leading SEOF mode at the pressure level and position of the maximum absolute value of the mode. As expected, the result closely resembles the two-dimensional structure identified in Fig. 4 with a slightly different period due to the different sampling of the data, see Table 1 . The fully three-dimensional structure is shown in Fig. 11 in terms of two surfaces at representative values of \pm 0.5 times the maximum absolute value of the leading mode. Here, the pressure levels are converted into altitude using the Standard Atmosphere. The quasiuniform zonal mean flow character of the QBO flow reversal in the equatorial stratosphere can be readily identified.
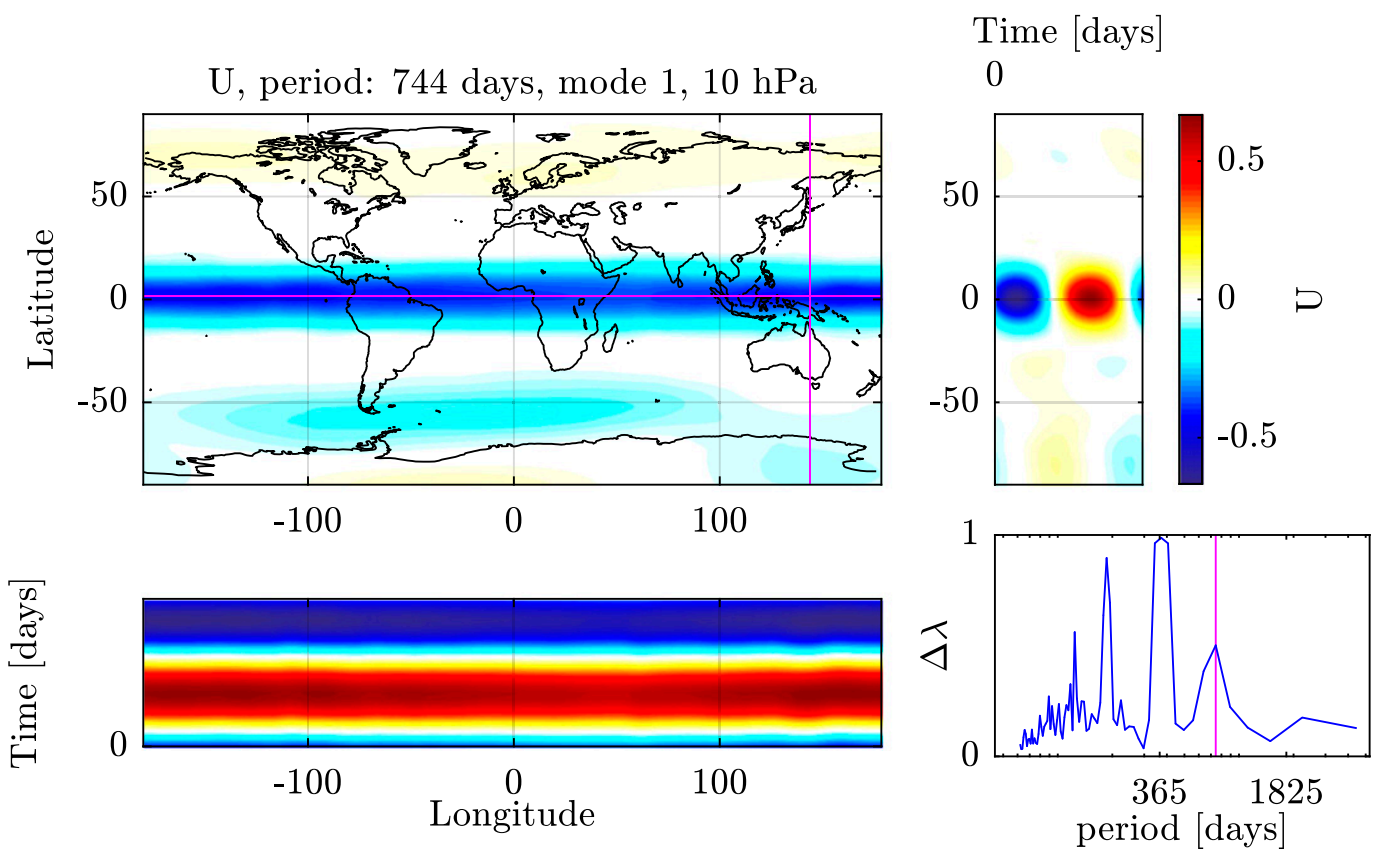

FIG. 10. As in Fig. 4, but for a slightly different period. 


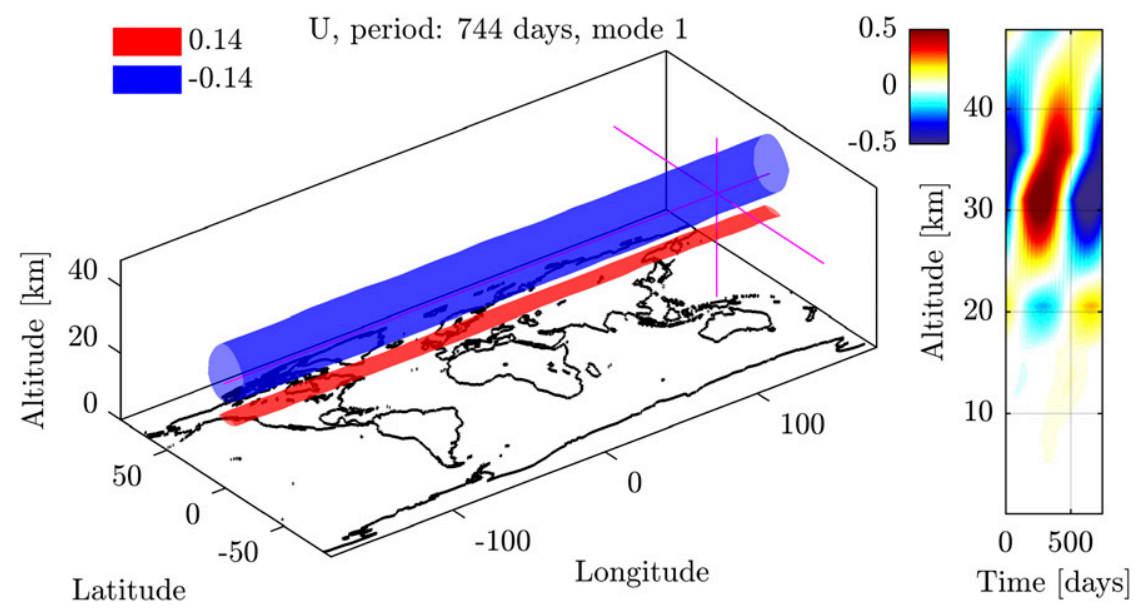

FIG. 11. (left) Real part of the leading three-dimensional SEOF mode for the horizontal velocity $U$ for a period of 744 days and (right) Hovmöller diagram showing the temporal variation of the leading mode over one period with altitude.

\section{Summary and conclusions}

The present study introduces spectral empirical orthogonal function (SEOF) analysis as a data-mining technique to educe climate patterns as temporal modes of variability from reanalysis data. In section 2, we first present the theoretical background of the method and its mathematical properties. We further contextualize SEOF by relating it to other popular modal decomposition techniques and operator-based forced linear system dynamics. In section 3, we illustrate SEOF analyses on a range of reanalysis data from the ERA Interim and ERA 20C databases. SEOF unambiguously identifies the ENSO and PDO, QBO and MJO from univariate twodimensional data. We furthermore apply the method to a bivariate ENSO index, and to MEI, a standard multivariate ENSO index that uses six different fields of surface data. Application to the MEI reveals intervariable cross correlations that link, for example, the equatorial oscillations of the sea surface temperature to wind patterns over West Antarctica. Similarly, three-dimensional modes of variability that are correlated in longitude, latitude, and altitude can be extracted. As an example, the QBO is educed from three-dimensional wind data over eight pressure levels.

Our results show that the SEOF analysis is well suited for data-mining large sets of weather or climate data for regional patterns and telecommunications. The SEOF spectrum, in particular, offers a means of identifying persistent features in an automated fashion. The interpretation of the results further benefits from the coherence property of the spatiotemporal modes and the direct link to the resolvent operator. The latter link implies that the dynamics of any pattern that is associated with a large separation between the first and the second SEOF eigenvalues can be modeled as a stochastically forced linear dynamical system.

In future research, we plan to explore the ENM approach in the context of SEOF. In particular, we speculate that SEOF based on wave activities can improve convergence of SEOF analysis and aid the physical interpretation of the results. Another important step is the incorporation of a null hypothesis based on a mean background spectrum, possibly red noise, into the analysis. Monte Carlo techniques as introduced by Allen and Smith (1996) [see also Groth and Ghil (2015)] for the closely related SSA method seem to offer a viable way forward in this respect. Alternatively, the background spectrum could be estimated from the data by filtering in the frequency domain and subsequently be removed from the Fourier realizations prior to the formation of the CSD matrix. This strategy was suggested by Wheeler and Kiladis (1999) with the goal of isolating significant spectral peaks in frequency-wavenumber diagrams. Also of eminent interest is the construction of SEOFbased stochastic models with predictive capabilities, as suggested early by North (1984) and demonstrated by Chekroun and Kondrashov (2017) and Kondrashov et al. (2018) in the context of DAH.

The MATLAB code used to conduct the SEOF analysis and the MARS request scripts to download the ECMWF reanalysis data is publicly available as a community resource.

\section{REFERENCES}

Allen, M. R., and L. A. Smith, 1996: Monte Carlo SSA: Detecting irregular oscillations in the presence of colored noise. J. Climate, 9, 3373-3404, https://doi.org/10.1175/1520-0442(1996) 009<3373:MCSDIO>2.0.CO;2. 
Alvera-Azcárate, A., A. Barth, M. Rixen, and J.-M. Beckers, 2005: Reconstruction of incomplete oceanographic data sets using empirical orthogonal functions: Application to the Adriatic Sea surface temperature. Ocean Modell., 9, 325-346, https:// doi.org/10.1016/j.ocemod.2004.08.001.

Baldwin, M. P., and Coauthors, 2001: The quasi-biennial oscillation. Rev. Geophys., 39, 179-229, https://doi.org/10.1029/ 1999RG000073.

Barnett, T., 1978: Estimating variability of surface air temperature in the Northern Hemisphere. Mon. Wea. Rev., 106, 1353-1367, https://doi.org/10.1175/1520-0493(1978) $106<1353$ :EVOSAT $>2.0 . \mathrm{CO} ; 2$.

__ 1999: Comparison of near-surface air temperature variability in 11 coupled global climate models. J. Climate, $\mathbf{1 2}$, 511-518, https://doi.org/10.1175/1520-0442(1999)012<0511: CONSAT $>2.0 . \mathrm{CO} ; 2$.

Bierkens, M., and L. Van Beek, 2009: Seasonal predictability of European discharge: NAO and hydrological response time. J. Hydrometeor., 10, 953-968, https://doi.org/10.1175/ 2009JHM1034.1.

Brunet, G., 1994: Empirical normal-mode analysis of atmospheric data. J. Atmos. Sci., 51, 932-952, https://doi.org/10.1175/15200469(1994)051<0932:ENMAOA > 2.0.CO;2.

Byrne, N. J., T. G. Shepherd, T. Woollings, and R. A. Plumb, 2017: Nonstationarity in Southern Hemisphere climate variability associated with the seasonal breakdown of the stratospheric polar vortex. J. Climate, 30, 7125-7139, https://doi.org/10.1175/ JCLI-D-17-0097.1.

Chekroun, M. D., and D. Kondrashov, 2017: Data-adaptive harmonic spectra and multilayer Stuart-Landau models. Chaos, 27, 093110, https://doi.org/10.1063/1.4989400.

Clem, K. R., and R. L. Fogt, 2013: Varying roles of ENSO and SAM on the Antarctic Peninsula climate in austral spring. J. Geophys. Res. Atmos., 118, 11481-11 492, https://doi.org/ 10.1002/jgrd.50860

Davis, R. E., 1976: Predictability of sea surface temperature and sea level pressure anomalies over the North Pacific Ocean. J. Phys. Oceanogr., 6, 249-266, https://doi.org/10.1175/1520 0485(1976)006<0249:POSSTA > 2.0.CO;2.

Dee, D. P., and Coauthors, 2011: The ERA-Interim reanalysis: Configuration and performance of the data assimilation system. Quart. J. Roy. Meteor. Soc., 137, 553-597, https:// doi.org/10.1002/qj.828.

Deser, C., and M. L. Blackmon, 1995: On the relationship between tropical and North Pacific sea surface temperature variations. J. Climate, 8, 1677-1680, https://doi.org/10.1175/ 1520-0442(1995)008<1677:OTRBTA > 2.0.CO;2.

Farrell, B. F., and P. J. Ioannou, 1996: Generalized stability theory. Part I: Autonomous operators. J. Atmos. Sci., 53, 2025-2040, https:// doi.org/10.1175/1520-0469(1996)053<2025:GSTPIA >2.0.CO;2.

Gamage, N., and W. Blumen, 1993: Comparative analysis of low-level cold fronts: Wavelet, Fourier, and empirical orthogonal function decompositions. Mon. Wea. Rev., 121, 2867-2878, https://doi.org/ 10.1175/1520-0493(1993)121<2867:CAOLLC $>2.0$. CO;2.

Ghil, M., and Coauthors, 2002: Advanced spectral methods for climatic time series. Rev. Geophys., 40, 1-41, https://doi.org/ 10.1029/2000RG000092.

Gray, L. J., J. A. Anstey, Y. Kawatani, H. Lu, S. Osprey, and V. Schenzinger, 2018: Surface impacts of the quasi biennial oscillation. Atmos. Chem. Phys., 18, 8227-8247, https://doi.org/ 10.5194/acp-18-8227-2018.

Groth, A., and M. Ghil, 2015: Monte Carlo Singular Spectrum Analysis (SSA) revisited: Detecting oscillator clusters in multivariate datasets. J. Climate, 28, 7873-7893, https://doi.org/ 10.1175/JCLI-D-15-0100.1.

—, Y. Feliks, D. Kondrashov, and M. Ghil, 2017: Interannual variability in the North Atlantic Ocean's temperature field and its association with the wind stress forcing. J. Climate, $\mathbf{3 0}$, 2655-2678, https://doi.org/10.1175/JCLI-D-16-0370.1.

Hannachi, A., I. T. Jolliffe, and D. B. Stephenson, 2007: Empirical orthogonal functions and related techniques in atmospheric science: A review. Int. J. Climatol., 27, 1119-1152, https:// doi.org/10.1002/joc.1499.

Hartmann, D. L., and H. H. Hendon, 2007: Resolving an atmospheric enigma. Science, 318, 1731-1732, https://doi.org/10.1126/ science. 1152502 .

Hawkins, E., and R. Sutton, 2007: Variability of the Atlantic thermohaline circulation described by three-dimensional empirical orthogonal functions. Climate Dyn., 29, 745-762, https://doi.org/10.1007/s00382-007-0263-8.

Held, I. M., 1985: Pseudomomentum and the orthogonality of modes in shear flows. J. Atmos. Sci., 42, 2280-2288, https://doi.org/ 10.1175/1520-0469(1985)042<2280:PATOOM >2.0.CO;2.

Kawamura, R., 1994: A rotated EOF analysis of global sea surface temperature variability with interannual and interdecadal scales. J. Phys. Oceanogr., 24, 707-715, https://doi.org/10.1175/ 1520-0485(1994)024<0707:AREAOG >2.0.CO;2.

Kondrashov, D., M. D. Chekroun, X. Yuan, and M. Ghil, 2018: Data-adaptive harmonic decomposition and stochastic modeling of arctic sea ice. Advances in Nonlinear Geosciences, A. A. Tsonis, Ed., Springer, 179-205.

Kutzbach, J. E., 1967: Empirical eigenvectors of sea-level pressure, surface temperature, and precipitation complexes over North America. J. Appl. Meteor., 6, 791-802, https://doi.org/10.1175/ 1520-0450(1967)006<0791:EEOSLP > 2.0.CO;2.

Legler, D. M., 1983: Empirical orthogonal function analysis of wind vectors over the tropical Pacific region. Bull. Amer. Meteor. Soc., 64, 234-241, https://doi.org/10.1175/1520-0477(1983)064<0234: EOFAOW $>2.0 . \mathrm{CO} ; 2$.

Limpasuvan, V., and D. L. Hartmann, 2000: Wave-maintained annular modes of climate variability. J. Climate, 13, 44144429, https://doi.org/10.1175/1520-0442(2000)013<4414: WMAMOC $>2.0 . \mathrm{CO} ; 2$.

Lintner, B. R., 2002: Characterizing global $\mathrm{CO}_{2}$ interannual variability with empirical orthogonal function/principal component (EOF/PC) analysis. Geophys. Res. Lett., 29, 1921, https://doi.org/10.1029/2001GL014419.

Lorenz, E. N., 1956: Empirical orthogonal functions and statistical weather prediction. Statistical Forecasting Project Sci. Rep. 1, MIT, 52 pp., https://eapsweb.mit.edu/sites/default/ files/Empirical_Orthogonal_Functions_1956.pdf.

Lumley, J. L., 1970: Stochastic Tools in Turbulence. 1st ed. Academic Press, 208 pp.

Mac Veigh, J., B. Barnier, and C. Le Provost, 1987: Spectral and empirical orthogonal function analysis of four years of European Centre for Medium-Range Weather Forecast wind stress curl over the North Atlantic Ocean. J. Geophys. Res., 92, 13 141-13 152, https://doi.org/10.1029/JC092iC12p13141.

Mak, M., 1995: Orthogonal wavelet analysis: Interannual variability in the sea surface temperature. Bull. Amer. Meteor. Soc., 76, 2179-2186, https://doi.org/10.1175/1520-0477(1995) $076<2179$ :OWAIVI $>2.0 . \mathrm{CO} ; 2$.

Manolakis, D. G., D. Manolakis, V. K. Ingle, and S. M. Kogon, 2005: Statistical and Adaptive Signal Processing: Spectral Estimation, Signal Modeling, Adaptive Filtering and Array Processing. Artech House Publishers, 816 pp. 
Mantua, N. J., and S. R. Hare, 2002: The Pacific decadal oscillation. J. Oceanogr., 58, 35-44, https://doi.org/10.1023/A:1015820616384.

Marshall, G. J., 2003: Trends in the Southern Annular Mode from observations and reanalyses. J. Climate, 16, 4134-4143, https:// doi.org/10.1175/1520-0442(2003)016<4134:TITSAM>2.0.CO;2.

Martinez, Y., G. Brunet, M. K. Yau, and X. Wang, 2011: On the dynamics of concentric eyewall genesis: Space-time empirical normal modes diagnosis. J. Atmos. Sci., 68, 457-476, https:// doi.org/10.1175/2010JAS3501.1.

Matsuo, T., and J. M. Forbes, 2010: Principal modes of thermospheric density variability: Empirical orthogonal function analysis of CHAMP 2001-2008 data. J. Geophys. Res., 115, A07309, https://doi.org/10.1029/2009JA015109.

Miller, J. K., and R. G. Dean, 2007a: Shoreline variability via empirical orthogonal function analysis: Part I temporal and spatial characteristics. Coastal Eng., 54, 111-131, https://doi.org/ 10.1016/j.coastaleng.2006.08.013.

$\longrightarrow$, and,$- 2007 \mathrm{~b}$ : Shoreline variability via empirical orthogonal function analysis: Part II relationship to nearshore conditions. Coastal Eng., 54, 133-150, https://doi.org/10.1016/ j.coastaleng.2006.08.014.

Min, S.-K., X. Zhang, F. W. Zwiers, and G. C. Hegerl, 2011: Human contribution to more-intense precipitation extremes. Nature, 470, 378-381, https://doi.org/10.1038/nature09763.

Mo, K. C., 2000: Relationships between low-frequency variability in the Southern Hemisphere and sea surface temperature anomalies. J. Climate, 13, 3599-3610, https://doi.org/10.1175/ 1520-0442(2000)013<3599:RBLFVI >2.0.CO;2.

Monahan, A. H., J. C. Fyfe, M. H. P. Ambaum, D. B. Stephenson, and G. R. North, 2009: Empirical orthogonal functions: The medium is the message. J. Climate, 22, 6501-6514, https:// doi.org/10.1175/2009JCLI3062.1.

Mu, Q., C. S. Jackson, and P. L. Stoffa, 2004: A multivariate empirical-orthogonal-function-based measure of climate model performance. J. Geophys. Res., 109, D15101, https://doi.org/ 10.1029/2004JD004584.

Navarra, A., and V. Simoncini, 2010: A Guide to Empirical Orthogonal Functions for Climate Data Analysis. Springer Science \& Business Media, $151 \mathrm{pp}$.

Newman, M., G. P. Compo, and M. A. Alexander, 2003: ENSOforced variability of the Pacific decadal oscillation. J. Climate, 16, 3853-3857, https://doi.org/10.1175/1520-0442(2003)016<3853: EVOTPD $>2.0 . \mathrm{CO} ; 2$.

North, G. R., 1984: Empirical orthogonal functions and normal modes. J. Atmos. Sci., 41, 879-887, https://doi.org/10.1175/ 1520-0469(1984)041<0879:EOFANM>2.0.CO;2.

Obukhov, A., 1947: Statistically homogeneous fields on a sphere. Uspekhi Mat. Nauk, 2 (2), 196-198.

Palmer, T. N., and D. L. Anderson, 1994: The prospects for seasonal forecasting-A review paper. Quart. J. Roy. Meteor. Soc., 120, 755-793, https://doi.org/10.1002/ QJ.49712051802.

Paolo, F., L. Padman, H. Fricker, S. Adusumilli, S. Howard, and M. Siegfried, 2018: Response of Pacific-sector Antarctic ice shelves to the El Niño/Southern Oscillation. Nat. Geosci., 11, 121-126, https://doi.org/10.1038/s41561-017-0033-0.

Plaut, G., and R. Vautard, 1994: Spells of low-frequency oscillations and weather regimes in the Northern Hemisphere. J. Atmos. Sci., 51, 210-236, https://doi.org/10.1175/1520-0469(1994)051<0210: SOLFOA $>2.0 . \mathrm{CO} ; 2$.

Poli, P., and Coauthors, 2016: ERA-20C: An atmospheric reanalysis of the twentieth century. J. Climate, 29, 4083-4097, https://doi.org/10.1175/JCLI-D-15-0556.1.
Pritchard, M. S., and R. C. Somerville, 2009: Empirical orthogonal function analysis of the diurnal cycle of precipitation in a multi-scale climate model. Geophys. Res. Lett., 36, L05812, https://doi.org/10.1029/2008GL036964.

Sardeshmukh, P. D., and P. Sura, 2009: Reconciling non-Gaussian climate statistics with linear dynamics. J. Climate, 22, 1193-1207, https://doi.org/10.1175/2008JCLI2358.1.

Schmidt, E., 1907: Zur theorie der linearen und nicht linearen integralgleichungen zweite abhandlung. Math. Ann., 64, 161-174, https://doi.org/10.1007/BF01449890.

Schmidt, O. T., and A. Towne, 2019: An efficient streaming algorithm for spectral proper orthogonal decomposition. Comput. Phys. Commun., 237, 98-109, https://doi.org/ 10.1016/j.cpc.2018.11.009.

,-- , G. Rigas, T. Colonius, and G. A. Brès, 2018: Spectral analysis of jet turbulence. J. Fluid Mech., 855, 953-982, https:// doi.org/10.1017/jfm.2018.675.

Servain, J., and D. M. Legler, 1986: Empirical orthogonal function analyses of tropical Atlantic sea surface temperature and wind stress: 1964-1979. J. Geophys. Res., 91, 14181-14191, https:// doi.org/10.1029/JC091iC12p14181.

Sirovich, L., 1987: Turbulence and the dynamics of coherent structures. Quart. Appl. Math., 45, 561-571, https://doi.org/ 10.1090/qam/910462.

_ scientific datasets. Int. J. Supercomput. Appl., 6, 50-68, https:// doi.org/10.1177/109434209200600104.

Smith, T. M., R. W. Reynolds, R. E. Livezey, and D. C. Stokes, 1996: Reconstruction of historical sea surface temperatures using empirical orthogonal functions. J. Climate, 9, 1403-1420, https:// doi.org/10.1175/1520-0442(1996)009<1403:ROHSST>2.0.CO;2.

Thompson, D. W., and J. M. Wallace, 2000: Annular modes in the extratropical circulation. Part I: Month-to-month variability. J. Climate, 13, 1000-1016, https://doi.org/10.1175/ 1520-0442(2000)013<1000:AMITEC > 2.0.CO;2.

Timmermann, A., and Coauthors, 2018: El Niño-Southern Oscillation complexity. Nature, 559, 535-545, https://doi.org/ 10.1038/s41586-018-0252-6.

Towne, A., O. T. Schmidt, and T. Colonius, 2018: Spectral proper orthogonal decomposition and its relationship to dynamic mode decomposition and resolvent analysis. J. Fluid Mech., 847, 821-867, https://doi.org/10.1017/jfm.2018.283.

Trenberth, K. E., 1997: The definition of El Niño. Bull. Amer. Meteor. Soc., 78, 2771-2777, https://doi.org/10.1175/15200477(1997)078<2771:TDOENO>2.0.CO;2.

Wang, B., and S.-I. An, 2005: A method for detecting seasondependent modes of climate variability: S-EOF analysis. Geophys. Res. Lett., 32, L15710, https://doi.org/10.1029/ 2005GL022709.

_ rainfall predictability: A predictable mode analysis. Climate Dyn., 44, 61-74, https://doi.org/10.1007/s00382-014-2218-1.

Wang, S., J. Huang, Y. He, and Y. Guan, 2014: Combined effects of the Pacific decadal oscillation and El Nino-Southern Oscillation on global land dry-wet changes. Sci. Rep., 4, 6651, https:// doi.org/10.1038/srep06651.

Weare, B. C., and R. Newell, 1977: Empirical orthogonal analysis of Atlantic Ocean surface temperatures. Quart. J. Roy. Meteor. Soc., 103, 467-478, https://doi.org/10.1002/qj.49710343707.

, and J. S. Nasstrom, 1982: Examples of extended empirical orthogonal function analyses. Mon. Wea. Rev., 110, 481485, https://doi.org/10.1175/1520-0493(1982)110<0481: EOEEOF $>2.0 . \mathrm{CO} ; 2$. 
_ A. R. Navato, and R. E. Newell, 1976: Empirical orthogonal analysis of Pacific sea surface temperatures. J. Phys. Oceanogr., 6, 671-678, https://doi.org/10.1175/1520-0485(1976) $006<0671:$ EOAOPS $>2.0 . \mathrm{CO} ; 2$.

Welch, P., 1967: The use of fast Fourier transform for the estimation of power spectra: A method based on time averaging over short, modified periodograms. IEEE Trans. Audio Electroacoust., 15, 70-73, https://doi.org/10.1109/TAU.1967.1161901.

Wheeler, M., and G. N. Kiladis, 1999: Convectively coupled equatorial waves: Analysis of clouds and temperature in the wavenumber-frequency domain. J. Atmos. Sci., 56, 374-399, https://doi.org/10.1175/1520-0469(1999)056<0374:CCEWAO> 2.0.CO;2.

Wolter, K., and M. S. Timlin, 1993: Monitoring ENSO in COADS with a seasonally adjusted principal component index. Proc. 17th Climate Diagnostics Workshop, Norman, OK, CIMMS, 52-57.

Wu, Z., B. Wang, J. Li, and F.-F. Jin, 2009: An empirical seasonal prediction model of the East Asian summer monsoon using ENSO and NAO. J. Geophys. Res., 114, D18120, https:// doi.org/10.1029/2009JD011733.

Zadra, A., G. Brunet, and J. Derome, 2002: An empirical normal mode diagnostic algorithm applied to NCEP reanalyses. J. Atmos. Sci., 59, 2811-2829, https://doi.org/10.1175/15200469(2002)059<2811:AENMDA > 2.0.CO;2.

Zhang, C., 2005: Madden-Julian Oscillation. Rev. Geophys., 43, RG2003, https://doi.org/10.1029/2004RG000158.

Zhang, Y., J. M. Wallace, and D. S. Battisti, 1997: ENSO-like interdecadal variability: 1900-93. J. Climate, 10, 1004-1020, https:// doi.org/10.1175/1520-0442(1997)010<1004:ELIV>2.0.CO;2. 